\title{
Integrated Guidance and Control Method for the Interception of Maneuvering Hypersonic Vehicle Based on High Order Sliding Mode Approach
}

\author{
Kang Chen, ${ }^{1}$ Bin Fu, ${ }^{1}$ Yuening Ding, ${ }^{2}$ and Jie Yan ${ }^{1}$ \\ ${ }^{1}$ College of Astronautics, Northwestern Polytechnical University, Xian 710072, China \\ ${ }^{2}$ Flight Automation Control Research Institute, Xian 710065, China \\ Correspondence should be addressed to Bin Fu; binfu@mail.nwpu.edu.cn
}

Received 27 April 2015; Revised 26 July 2015; Accepted 29 July 2015

Academic Editor: Giuseppe Rega

Copyright ( $\odot 2015$ Kang Chen et al. This is an open access article distributed under the Creative Commons Attribution License, which permits unrestricted use, distribution, and reproduction in any medium, provided the original work is properly cited.

\begin{abstract}
This paper focuses on the integrated guidance and control (IGC) method applied in the interception of maneuvering near space hypersonic vehicles using the homogeneous high order sliding mode (HOSM) approach. The IGC model is derived by combining the target-missile relative motion and dynamic equations. Then, a fourth-order sliding mode controller is implemented in the augmented IGC model. To estimate the high order derivatives of the sliding manifold which is required in the HOSM method, an Arbitrary Order Robust Exact Differentiator is presented. At last, the idea of virtual control is introduced to alleviate the chattering of the control input without using any saturation functions which may lead to a loss of the robustness. And the stability of the closed-loop system with presented fourth-order homogeneous HOSM controller is also proved theoretically. Finally, simulation results are provided and analyzed to demonstrate the effectiveness of the proposed method in three typical engagement scenarios.
\end{abstract}

\section{Introduction}

Because of its speed advantage and flexible maneuverability, the hypersonic vehicle may certainly become a severe threat in the future battlefield; to deal with the threat, the research on the interception of the hypersonic vehicle is already on the agenda. Primarily, the interception of the hypersonic vehicle faces the following issues: (1) when the speed of its target is much faster than the interception missile, the effective attack area of the traditional guidance law greatly shrinks, and it is impossible to accomplish the tail-chase or backward interception; (2) at the high altitude of 25 to $40 \mathrm{~km}$ where the hypersonic vehicle flies, the air is relatively thin, the aerodynamic efficiency of an interception missile is low, and there is a limited usable overload for the interceptor; (3) it is difficult to destroy such a hypersonic target with the traditional destructive means such as near explosion fragments, requiring that the interception missile should use knock-on collision as much as possible to attack the target, namely, minimal target missing. As the requirements for guidance accuracy are higher, researchers do massive work to advance the guidance and control theory.

During the past decades, the proportional navigation (PN) guidance law is a popular and widely used method in missile interception missions for its ease of implementation and high efficiency. The principle of PN guidance law in [1] is that the commanded normal acceleration of the missile is proportional to the line-of-sight (LOS) rate, which is simple and effective under a wide range of engagement scenarios. However, the use of the PN guidance law is also limited; for example, as the distance between the missile and its target is closing or the target acts an unpredictable maneuver, the LOS rate may grow extremely fast, and, subsequently, the overload needed at the end phase also diverges at last. As for the overload autopilot accounting for a second-order dynamic, the real acceleration response of the missile to the fast-changing high-frequency overload command lags behind and attenuates and eventually caused a large miss distance. Therefore, many new guidance laws crop up. 
Being famous for its good robustness to bounded disturbances, the sliding mode control method is introduced into the study of guidance law. In [2] Zhou et al. gave the conditions for the sliding mode motion of a linear time-varying system not to be disturbed by the disturbances and parameter perturbations and proposed an adaptive sliding mode guidance law (ASMG); simulation results demonstrated that the ASMG is robust to uncertainties like target acceleration. To get finite time convergence, modified SMC like terminal sliding mode control (TSMC) is proposed. In [3] Zeng and $\mathrm{Hu}$ combine the advantages of linear and terminal sliding mode controls which guarantee the convergence of tracking errors in finite time. And then, nonsingular terminal sliding mode control (NTSMC) is introduced in the guidance law design. In [4] Kumar et al. proposed a nonsingular terminal sliding mode guidance law with finite time convergence, which avoids the singularity that may lead to the saturation of the control. Integral SMC (ISMC) like that introduced in [5] is another revised sliding mode control which introduced the integral sliding mode scheme to the guidance law design; the proposed ISMC guidance law provided a smaller control magnitude than the traditional sliding mode design. The advance of the $H_{\infty}$ controller greatly enriched the methods to implement a robust control system. In [6] Savkin et al. suitably modified the $H_{\infty}$ control theory and provided an effective framework for the precision missile guidance problem which showed much better performance than the linear quadratic optimal guidance law in the computer simulation. In [7], Chen and Yang proposed a mixed $H_{2} / H_{\infty}$ guidance design against maneuvering targets, in which the complete nonlinear kinematics of the pursuit-evasion motion was considered.

In addition to the separated guidance and control law, the integrated guidance and control method is under heated discussion. According to the relative motion between the target and the missile, the traditional guidance law calculates the overload needed to hit the target and inputs the overload command into the overload autopilot, while the integrated guidance and control (IGC) method gives the rudder deflection command directly to the missile according to the relative motion, which evidently responds more quickly. In [8] Menon et al. designed an IGC method by using the linear quadratic optimal theory, but the robustness of the system is not satisfactory. In [9] Vaddi et al. provided a fully numerical methodology for deriving state-dependent Riccati equation controllers for arbitrarily complex dynamic systems and applied it in the IGC design of a missile. Simulation results demonstrated the effectiveness of the method. In [10] Xin et al. employed the theta-D method to give an approximate closed-form suboptimal feedback controller to the nonlinear infinite-horizon IGC problem. Taking another look, sliding mode approaches are also employed in the IGC design of homing missiles. In [11], Shima et al. proposed the sliding mode integrated guidance and control method based on the ZEM. Shtessel and Tournes does massive research on the high order sliding mode controller design and proposed his new method. In [12], he designed the high order sliding mode guidance law based on the smooth second-order sliding mode control (SSOSMC) which is smoother in high orders than the traditional second-order sliding mode guidance law. In [13], based on the geometrical homogeneity theory, Dong et al. designed the tranquility control law for the integrated guidance and control model, which, however, has rather more parameters and is sensitive to parameters and the parameters must be carefully selected. In [14], Mingzhe and Guangren used the sliding mode control theory to design the adaptive nonlinear feedback controller, which is primarily for fixed ground target, being unable to deal with the vehement perturbation caused by target maneuvering.

Motivated by the aforementioned considerations, this work will design an IGC scheme for the interception of the near space maneuvering hypersonic vehicles. Firstly, a lineof-sight (LOS) rate feedback scheme is adopted to derive the IGC law and as the relative order of the control input to the LOS rate is higher than one, a HOSM approach is introduced. Secondly, to implement the HOSM approach, the $n$ times derivations of the sliding manifold $\dot{S}, \ddot{S}, \dddot{S}, \ldots, S^{(n)}$ must be known. However, reconstruction of each $\dot{S}, \ddot{S}, \ddot{S}, \ldots, S^{(n)}$ by their analytical expression could be rather difficult in practice. An alternative way adopted in this paper is to use the Arbitrary-Order Robust Exact Differentiator (AORED) to estimate the derivations. And next, to alleviate the chattering phenomenon caused by the HOSM controller the idea of virtual control is introduced. The virtual control $v$ is designed and used as the control input of a system extended from the original one, and the real control $u$ acting on the real system is obtained by integrating the virtual control $v$. Benefitting from the integration element, the real control input $u$ could be smooth enough for the implementation without reducing the robustness of the HOSM method. Finally, the proposed method is implemented in a 3-dof model.

The remaining part of this paper is organized as follows. In Section 2 the IGC model is derived in the longitudinal plane. In Section 3, the quasi-continuous HOSM controller as well as the AORED is designed. In Section 4, the baseline separated guidance laws and controller are given. In Section 5, the numerical simulations are demonstrated in three typical engagement scenarios. And conclusions are made in Section 6.

\section{Integrated Guidance and Control Model}

The traditional guidance and control algorithm usually uses the guidance loop as its outer loop and is only responsible for giving commanded overload; then the control loop is only responsible for tracking the overload command, eventually achieving the missile's guidance toward its target. Although it is always desirable to design the control loop or the autopilot, to have better dynamic performance, in actuality, the controller always has some delay and attenuation. As a result, the missile always has some error in acting the overload command. That is one of the reasons why an interceptor misses its target.

The integrated guidance and control algorithm combines the guidance and control loops into one loop and avoids the delay and attenuation caused by them. Its architecture is shown in Figure 1. 


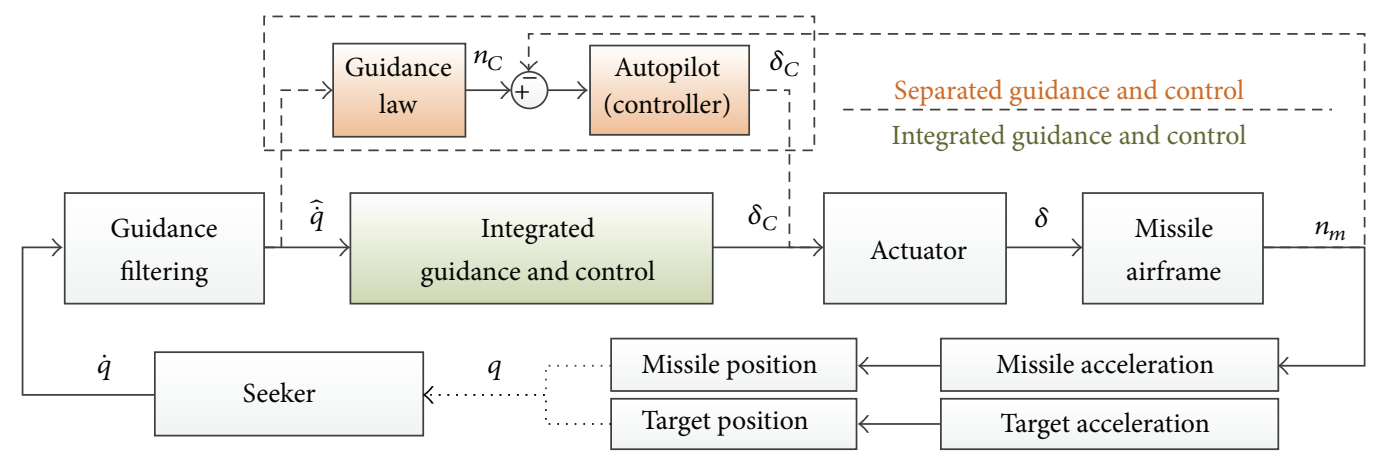

Figure 1: Integrated guidance and control.

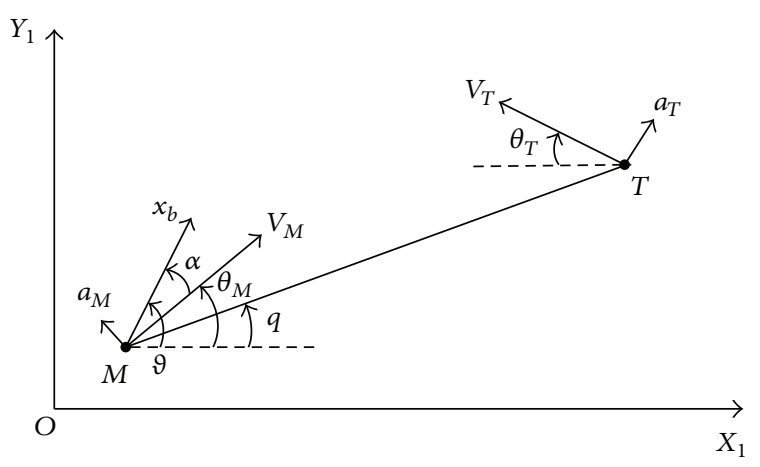

Figure 2: Engagement geometry.

2.1. The Engagement Dynamics. Without loss of generality, we present hereinafter only the subsystems that govern the motion of an interceptor in its longitudinal plane. The planar engagement between the interceptor and its target is shown in Figure 2.

The study of guidance laws usually regards the missile and its target as mass points, deliberates on the mass point of its rigid motion, and ignores the attitude of the missile airframe and changes in its attitude. By contrast, the integrated guidance and control method takes the dynamic characteristics of the missile airframe into consideration; therefore, the derivation involves the pitch angle, pitch rate, and angle of attack of the missile, as shown in Figure 2.

The motion equations of the missile in the longitudinal plane are as follows:

$$
\begin{aligned}
\frac{d V_{M}}{d t} & =\frac{1}{m}\left(P \cos \alpha-X-m g \sin \theta_{M}\right), \\
\frac{d \theta_{M}}{d t} & =\frac{1}{m V_{M}}\left(P \sin \alpha+Y-m g \cos \theta_{M}\right), \\
\frac{d x_{M}}{d t} & =V_{M} \cos \theta_{M} \\
\frac{d y_{M}}{d t} & =V_{M} \sin \theta_{M} \\
\frac{d \omega_{Z}}{d t} & =\frac{M_{Z}}{J_{Z}}
\end{aligned}
$$

$$
\begin{aligned}
\frac{d \vartheta}{d t} & =\omega_{Z}, \\
\alpha & =\vartheta-\theta,
\end{aligned}
$$

where $\left(x_{M}, y_{M}\right)$ is the position, $m$ is the mass, $X$ is the axial force, $P$ is the thrust force, the missile's velocity is $V_{M}$, and its flight path angle is $\theta_{M}$; the LOS angle between the missile and its target is $q$; the relative distance between the missile and its target is $r$, the velocity of the target is $V_{T}$, and its flight path angle is $\theta_{T} ; x_{b}$ is the axle of the missile's airframe; the pitch angle is $\vartheta$; the angle of attack is $\alpha$.

The motion equations of the target are as follows:

$$
\begin{aligned}
& \frac{d x_{T}}{d t}=-V_{T} \cos \theta_{T}, \\
& \frac{d y_{T}}{d t}=V_{T} \sin \theta_{T}, \\
& \frac{d \theta_{T}}{d t}=\frac{a_{T N}}{V_{T}} .
\end{aligned}
$$

The interception is characterized by two variables, namely, the target range and the LOS angle. The kinematic equations are expressed by the following relations:

$$
\begin{array}{r}
\frac{d r}{d t}=-V_{M} \cos \left(\theta_{M}-q\right)-V_{T} \cos \left(\theta_{T}+q\right), \\
r \frac{d q}{d t}=-V_{M} \sin \left(\theta_{M}-q\right)+V_{T} \sin \left(\theta_{T}+q\right) .
\end{array}
$$

2.2. The Model Simplification. To simplify the model and make further derivations, we make the following two assumptions.

Assumption 1. Within the terminal phase of the interception, the missile has no thrust and its gravity is not taken into account.

Assumption 2. Within the terminal phase of the interception, the missile's speed does not change. 
Then (1) and (2) can be reformed as below:

$$
\begin{aligned}
& \frac{d V_{M}}{d t}=0, \\
& \frac{d \theta_{M}}{d t}=\frac{Y}{m V_{M}} .
\end{aligned}
$$

Denote that $a_{M N}=Y / m ; Y$ is the normal force; $a_{M N}$, $a_{T N}$ are the normal acceleration of the missile and target, respectively, and then

$$
\begin{gathered}
\dot{\theta}_{M}=\frac{a_{M N}}{V_{M}}, \\
\dot{\theta}_{T}=\frac{a_{T N}}{V_{T}} .
\end{gathered}
$$

The normal force $Y$ and the pitch moment $M_{Z}$ acting on the missile are usually expressed, respectively, as follows:

$$
\begin{gathered}
Y=C_{Y}^{\alpha} \alpha Q S_{\text {ref }}+C_{Y}^{\delta_{Z}} \delta_{Z} Q S_{\text {ref }}, \\
M_{Z}=m_{Z}^{\delta_{Z}} \delta_{Z} Q S_{\text {ref }} l+m_{Z}^{\alpha} \alpha Q S_{\text {ref }} l
\end{gathered}
$$

where $C_{Y}^{\alpha}$ is the coefficient of normal force caused by the angle of attack; $C_{Y}^{\delta_{Z}}$ is the coefficient of normal force caused by the rudder deflection angle $\delta_{Z} ; Q$ is the dynamic pressure; $S_{\text {ref }}$ is the reference area; $l$ is the reference length. But the normal force produced by the rudder deflection angle $\delta_{Z}$ is orders of magnitude smaller than that produced by the angle of attack, so (13) is simplified as

$$
Y=C_{Y}^{\alpha} \alpha Q S_{\text {ref }}
$$

Then

$$
a_{M N}=\frac{Y}{m}=\frac{C_{Y}^{\alpha} \alpha Q S_{\text {ref }}}{m} .
$$

Then the dynamic equations can be simplified as

$$
\begin{aligned}
\frac{d \theta_{M}}{d t} & =\frac{C_{Y}^{\alpha} \alpha Q S_{\text {ref }}}{m V_{M}}, \\
\frac{d \omega_{Z}}{d t} & =\frac{m_{Z}^{\delta_{Z}} \delta_{Z} Q S_{\mathrm{ref}} l+m_{Z}^{\alpha} \alpha Q S_{\mathrm{ref}} l}{J_{Z}}, \\
\frac{d \vartheta}{d t} & =\omega_{Z}, \\
\alpha & =\vartheta-\theta_{M} .
\end{aligned}
$$

2.3. The Integrated Guidance and Control Model. Following the above derivation, we select the state variables as $\left(\dot{q}, \theta_{M}, \alpha, \omega_{Z}\right)^{T}$ and obtain the following nonlinear integrated guidance and control model:

$$
\begin{aligned}
\ddot{q}= & -2 \frac{\dot{r}}{r} \cdot \dot{q}-\frac{C_{Y}^{\alpha} Q S_{\mathrm{ref}}}{m r} \cos \left(\theta_{M}-q\right) \cdot \alpha \\
& +\frac{a_{T N}}{r} \cos \left(\theta_{T}+q\right), \\
r \dot{q}= & -V_{M} \sin \left(\theta_{M}-q\right)+V_{T} \sin \left(\theta_{T}+q\right), \\
\dot{\theta}_{M}= & \frac{1}{m V_{M}} C_{Y}^{\alpha} Q S_{\mathrm{ref}} \cdot \alpha, \\
\dot{\alpha}= & \omega_{Z}-\frac{1}{m V_{M}} C_{Y}^{\alpha} Q S_{\mathrm{ref}} \cdot \alpha, \\
\dot{\omega}_{Z}= & \frac{m_{Z}^{\delta_{Z}} Q S_{\mathrm{ref}} l}{J_{Z}} \cdot \delta_{Z}+\frac{m_{Z}^{\alpha} Q S_{\mathrm{ref}} l}{J_{Z}} \alpha .
\end{aligned}
$$

2.4. The Relative Degree of Control Input. To obtain the relative degree of the control input $\delta$ of the integrated guidance and control method, we keep on deriving the LOS angular velocity $\dot{q}$ until the explicit formula of its derivative of a certain order contains the control input.

The derivation of (10) produces

$\ddot{q}$

$$
=\frac{1}{r}\left[-2 \dot{r} \dot{q}-a_{M N} \cos \left(\theta_{M}-q\right)+a_{T N} \cos \left(\theta_{T}+q\right)\right] .
$$

The LOS angular velocity is expressed as the derivative of the first order and does not contain the control input $\delta$ explicitly. The continuous derivation of the above equation produces

$$
\begin{aligned}
\dddot{q}= & \frac{1}{r}\left\{-2 \ddot{r} \dot{q}-3 \dot{r} \ddot{q}-\left[a_{M N}\left(\dot{q}-\dot{\theta}_{M}\right) \sin \left(\theta_{M}-q\right)\right.\right. \\
& \left.+\dot{a}_{M N} \cos \left(\theta_{M}-q\right)\right]-\left[a_{T N}\left(\dot{q}+\dot{\theta}_{T}\right) \sin \left(\theta_{T}+q\right)\right. \\
& \left.\left.-\dot{a}_{T N} \cos \left(\theta_{T}+q\right)\right]\right\},
\end{aligned}
$$

where the LOS angular rate is expressed as the differentiating of the second order, and $\dot{a}_{M}$ can be expressed as

$$
\begin{aligned}
\dot{a}_{M N} & =\frac{C_{Y}^{\alpha} Q S_{\text {ref }}}{m} \dot{\alpha}=\frac{C_{Y}^{\alpha} Q S_{\text {ref }}}{m}\left(\dot{\vartheta}-\dot{\theta}_{M}\right) \\
& =\frac{C_{Y}^{\alpha} Q S_{\text {ref }}}{m}\left(\omega_{Z}-\dot{\theta}_{M}\right) .
\end{aligned}
$$

Although the control volume $\delta_{Z}$ does not appear in the derivative of the second order, (18) shows that $\dot{\omega}_{Z}$ contains $\delta_{Z}$. We continue to derive (23) and substitute $\ddot{a}_{M}$ as follows:

$$
\begin{aligned}
\ddot{a}_{M N}= & \frac{C_{Y}^{\alpha} Q S_{\text {ref }}}{m}\left(\dot{\omega}_{Z}-\frac{\dot{a}_{M}}{V_{M}}\right) \\
= & \frac{C_{Y}^{\alpha} Q S_{\text {ref }}}{m} \frac{m_{Z}^{\delta_{Z}} Q S_{\text {ref }} l_{\text {ref }}}{J_{Z}} \cdot \delta_{Z} \\
& +\frac{C_{Y}^{\alpha} Q S_{\text {ref }}}{m}\left(\frac{m_{Z}^{\alpha} Q S_{\text {ref }} l_{\text {ref }}}{J_{Z}} \alpha-\frac{\dot{a}_{M}}{V_{M}}\right) .
\end{aligned}
$$


Thus

$$
q^{(4)}=f_{\delta_{Z}} \cdot \delta_{Z}+\frac{1}{r}\left(f_{1}+f_{2}+f_{3}+f_{4}+f_{5}+f_{6}\right),
$$

where

$$
\begin{aligned}
& f_{\delta_{Z}}=-\frac{1}{r} \frac{C_{Y}^{\alpha} Q S_{\mathrm{ref}} \cos \eta_{M}}{m} \frac{m_{Z}^{\delta_{Z}} Q S_{\mathrm{ref}} l_{\mathrm{ref}}}{J_{Z}}, \\
& f_{1}=\left(\omega_{Z}-\dot{\theta}_{M}\right)\left(-2 \dot{\theta} \frac{a_{M N}}{\alpha} \sin \eta_{M}+\dot{q} \frac{a_{M N}}{\alpha} \sin \eta_{M}\right. \\
& \left.+\dot{\theta} \frac{a_{M N}}{\alpha^{2}} \cos \eta_{M}\right)-\frac{a_{M N} \cos \eta_{M}}{\alpha} \frac{m_{Z}^{\alpha} \alpha Q S_{\mathrm{ref}} l_{\mathrm{ref}}}{J_{Z}} \\
& f_{2}=\dot{\theta}_{M}\left(\left(\dot{\theta}_{M}-\dot{q}\right) a_{M N} \cos \eta_{M}\right. \\
& \left.-\left(\omega_{Z}-\dot{\theta}_{M}\right) \frac{a_{M N}}{\alpha} \sin \eta_{M}\right) \\
& f_{3}=\dot{q}\left(-\dot{\theta}_{M} a_{M N} \cos \eta_{M}\right. \\
& +\dot{q}\left(a_{M N} \cos \eta_{M}-a_{T N} \cos \eta_{T}\right)-\dot{\theta}_{T} a_{T N} \cos \eta_{T} \\
& \left.+\frac{a_{M N}}{\alpha}\left(\omega_{Z}-\dot{\theta}_{M}\right) \sin \eta_{M}-\dot{a}_{T} \sin \eta_{T}\right), \\
& f_{4}=\dot{\theta}_{T}\left(-\dot{q} a_{T} \cos \eta_{T}-\dot{\theta}_{T} a_{T} \cos \eta_{T}-\dot{a}_{T} \sin \eta_{T}\right), \\
& f_{5}=\dot{a}_{T}\left(-\dot{q} \sin \eta_{T}-2 \dot{\theta}_{T} \sin \eta_{T}\right)+\ddot{a}_{T} \cos \eta_{T}, \\
& f_{6}=\ddot{q}\left(a_{M N} \sin \eta_{M}-a_{T} \sin \eta_{T}-2 \ddot{r}\right)-4 \dot{r} \cdot \ddot{q}-3 \ddot{r} \cdot \ddot{q} \\
& -2 \dddot{r} \cdot \dot{q} \\
& \eta_{M}=\theta_{M}-q \text {, } \\
& \eta_{T}=\theta_{T}+q .
\end{aligned}
$$

In (26), it can be seen that the control input $\delta_{Z}$ appears expressly in the third-order derivative of the control output $\dot{q}$. Therefore, the relative degree of the control input $\delta_{Z}$ is 3 .

\section{The Quasi-Continuous High Order Sliding Mode Controller}

3.1. Sliding Mode Manifold Design. To design the HOSM controller, a sliding manifold must be chosen first. In this design, we try to make the LOS rate converge to zero or a small neighbor domain near zero, thus ensuring that the missile approaches its target in a quasi-parallel way which will lead to a minimal overload requirement. So the sliding manifold is chosen as follows:

$$
\sigma=\dot{q}
$$

From the above discussion in Section 2, we know that the control input in relation to control output $\dot{q}$, namely, the relative degree of sliding mode manifold $\sigma$, is 3 . So the following design will be about a third-order sliding mode controller.
3.2. Design of the Quasi-Continuous HOSM Controller. First, (26) can be expressed as follows:

$$
\dddot{\sigma}=h(t, x)+g(t, x) u,
$$

where $h(t, x), g(t, x)$, and $u$ are expressed as follows:

$$
\begin{aligned}
h(t, x) & =\frac{1}{r}\left(f_{1}+f_{2}+f_{3}+f_{4}+f_{5}+f_{6}\right), \\
g(t, x) & =f_{\delta_{Z}}, \\
u & =\delta_{Z} .
\end{aligned}
$$

According to the quasi-continuous high order sliding mode control method proposed by Levant in [15], the sliding mode manifold whose relative degree is 3 should be designed in the following form, where $\beta$ is a control gain term:

$$
u=-\beta \frac{\ddot{\sigma}+2\left(|\dot{\sigma}|+|\sigma|^{2 / 3}\right)^{-1 / 2}\left(\dot{\sigma}+|\sigma|^{2 / 3} \operatorname{sign} \sigma\right)}{|\ddot{\sigma}|+2\left(|\dot{\sigma}|+|\sigma|^{2 / 3}\right)^{1 / 2}} .
$$

The conditions under which the LOS angular velocity may converge are as follows:

$$
\begin{aligned}
0 & <K_{m} \leq g(t, x) \leq K_{M}, \\
|h(t, x)| & \leq C
\end{aligned}
$$

where $K_{m}, K_{M}$, and $C$ are all larger than zero. This is a proven theorem by Levant in [15].

The system we discussed meets the above requirements and the proof is as follows.

Equation (27) shows the following:

$$
\begin{aligned}
g(t, x) & =f_{\delta_{Z}} \\
& =-\frac{1}{r} \frac{C_{Y}^{\alpha} Q S_{\mathrm{ref}}}{m} \frac{m_{z}^{\delta Z} Q S_{\mathrm{ref}} l_{\mathrm{ref}}}{J_{Z}} \cos \left(\theta_{M}-q\right) .
\end{aligned}
$$

The dynamic pressure $Q$ is $Q=\rho V_{M}^{2} / 2$, where $\rho=$ $0.08803 \mathrm{Kg} / \mathrm{m}^{3}$ (altitude $=20 \mathrm{Km}$ ) is the air density and $V_{M}=$ $2000 \mathrm{~m} / \mathrm{s}$ is the speed of the missile, so $Q$ is always positive.

$S_{\text {ref }}=0.26 \mathrm{~m}^{2}$ and $l_{\text {ref }}=3.65 \mathrm{~m}$ denote the reference area and the reference length of the missile; they are both positive constant.

$m=100 \mathrm{Kg}$ denotes the missile mass. $J_{Z}=106 \mathrm{~m}^{2} \mathrm{Kg}$ denotes the rotational inertia.

$r$ is the relative distance; it is always a positive number.

$C_{Y}^{\alpha}$ is the lift coefficient caused by the angle of attack, it varies from 0.18 to 0.37 , and it is always a positive number.

$m_{z}^{\delta z}$ is the moment coefficient caused by the actuator deflection. In the normal layout (actuator lays behind the center of gravity) $m_{z}^{\delta z}$ is always negative.

Meanwhile, consider that the missile under guidance and control is unlikely to fly away from its target; namely, the angle between the missile's velocity and its LOS direction cannot be larger than $90^{\circ}$; then

$$
\begin{aligned}
\left|\theta_{M}-q\right| & <\frac{\pi}{2} \\
& \Longrightarrow \cos \left(\theta_{M}-q\right)>0 .
\end{aligned}
$$


Summing up the above conditions, then we can get

$$
g(t, x)>0
$$

In other words, there is a positive real number $K_{m}$ existing that could satisfy the following condition:

$$
0<K_{m}<g(t, x) .
$$

Before the missile hits on the target, the term will be positive and limited; then, we can get

$$
0<K_{m}<g(t, x)<K_{M}
$$

With (37), then

$$
h(t, x)=\frac{1}{r}\left(f_{1}+f_{2}+f_{3}+f_{4}+f_{5}+f_{6}\right) .
$$

In the practice sense, the changes in both the LOS rate and the acceleration of the missile and the acceleration of the target are limited and continuous. So the following variables $\dot{q}, \ddot{q}, \ddot{q}, \dot{a}_{M}, \dot{a}_{T}$, and $\ddot{a}_{T}$ are all bounded. However, because $h(t, x)$ contains the item $1 / r$, when the relative distance between the missile and its target is zero, the boundary of $h(t, x)$ is not guaranteed. In [15], Levant only requires that condition (39) should be locally valid, not requiring that it should be globally valid. Therefore, the integrated guidance and control method is applicable here. So, the above mentioned condition is satisfied with a positive number $C$ :

$$
|h(t, x)| \leq C
$$

3.3. The Virtual Control Design. When using the sliding mode control method, the avoidance of the chattering phenomenon has always been a key issue being discussed. In the traditional method, researchers in $[16,17]$ have proposed several saturation functions to replace the sign functions to build a boundary layer to alleviate the chattering or to use fuzzy logic to displace the high-frequency switching term. To our knowledge, none of these approaches has proven that the refined controller still retains their robustness against the uncertainties and disturbances. In this work, in order to alleviate the chattering phenomenon, we do not directly use the third-order controller but introduce the virtual control $u_{i}=\dot{\delta}_{Z}$ to perform the actual control:

$$
\delta_{Z}=\int \dot{\delta}_{Z} \mathrm{dt}=\int u_{i} \mathrm{dt} .
$$

After the relative degree is increased to the fourth order, we get the following expressions:

$$
\begin{aligned}
& \sigma^{(4)}=h^{*}(t, x)+g^{*}(t, x) u_{i}=h^{*}(t, x)+g(t, x) u_{i}, \\
& h^{*}(t, x)=\dot{h}(t, x)+\dot{g}(t, x) \delta_{Z}, \\
& \dot{g}(t, x) \\
& \quad=\frac{m_{z}^{\delta_{Z}} Q^{2} S^{2} l C_{Y}^{\alpha}}{J_{Z} m r^{2}}\left[\left(\dot{q}-\dot{\theta}_{M}\right) r \sin \eta_{M}+\dot{r} \cos \eta_{M}\right] .
\end{aligned}
$$

Even though the expression of $\dot{h}(t, x)$ is rather complicated, it is still the function of $\dot{q}, \ddot{q}, \ddot{q}, \dot{a}_{M}, \dot{a}_{T}$, and $\ddot{a}_{T}$; therefore, similar to $h(t, x)$, it has its boundary except the moment when the missile hits on its target. For the same reason, $\dot{g}(t, x)$ and the rudder deflection $\delta_{Z}$ also have their boundaries. Therefore we get the condition that $\left|h^{*}(t, x)\right| \leq C,(C>0)$. Because $\dot{\delta}_{Z}$ is obtained through the derivation of $\delta_{Z}, g^{*}(t, x)$ is the same as $g(t, x)$; thus $0<K_{m}<g^{*}(t, x)<K_{M}$ is satisfied.

According to the formula of the fourth-order controller given by Levant in [15], we give the following formulae for the virtual control $u_{i}$ :

$$
\begin{aligned}
u_{i} & =-\beta \frac{\Phi_{3,4}}{N_{3,4}}, \\
\Phi_{3,4} & =\ddot{\sigma}+3[|\ddot{\sigma}| \\
& \left.+\left.\left(|\dot{\sigma}|+0.5|\sigma|^{3 / 4}\right)^{-1 / 3}|\dot{\sigma}+0.5| \sigma\right|^{3 / 4} \operatorname{sign} \sigma \mid\right]^{-1 / 2} \\
& \cdot[\ddot{\sigma} \\
& \left.+\left.\left(|\dot{\sigma}|+0.5|\sigma|^{3 / 4}\right)^{-1 / 3}|\dot{\sigma}+0.5| \sigma\right|^{3 / 4} \operatorname{sign} \sigma \mid\right], \\
N_{3,4} & =|\ddot{\sigma}|+3[|\ddot{\sigma}| \\
& \left.+\left.\left(|\dot{\sigma}|+0.5|\sigma|^{3 / 4}\right)^{-1 / 3}|\dot{\sigma}+0.5| \sigma\right|^{3 / 4} \operatorname{sign} \sigma \mid\right]^{-1 / 2} \\
& +\mid \ddot{\sigma} \\
& +\left(|\dot{\sigma}|+0.5|\sigma|^{3 / 4}\right)^{-1 / 3}\left(\dot{\sigma}+0.5|\sigma|^{3 / 4} \operatorname{sign} \sigma\right) \mid .
\end{aligned}
$$

The integral of the virtual control $\dot{\delta}_{Z}$ produces the actual control command $\delta_{Z} . \ddot{\sigma}$ in the controller is obtained with the Arbitrary-Order Robust Exact Differentiator presented in the following section.

3.4. The Arbitrary-Order Robust Exact Differentiator. The quasi-continuous HOSM control method needs to use the third derivative of the sliding manifold, namely, $q^{(4)}$. How to calculate or accurately estimate $q^{(4)}$ is one of the key problems to be solved. We use the Arbitrary-Order Robust Exact Differentiator designed by Levant to differentiate the LOS rate $\dot{q}$, thus obtaining $\ddot{q}, \ddot{q}$, and $q^{(4)}$. valid:

According to (44) and (45), the following condition is

$$
|\dddot{\sigma}| \leq C+\beta K_{M}
$$

The Arbitrary-Order Robust Exact Differentiator can be constructed in accordance with high order sliding modes, differentiation, and output feedback control in [18].

If a certain signal $f(t)$ is a function consisting of a bounded Lebesgue-measurable noise with unknown base 
signal $f_{0}(t)$ whose $r$ th derivative has a known Lipschitz constant $L>0$, then the $n$ th-order differentiator is defined as follows:

$$
\begin{aligned}
& \dot{z}_{0}=v_{0} \text {, } \\
& v_{0}=-\lambda_{0} L^{1 /(n+1)}\left|z_{0}-f(t)\right|^{n /(n+1)} \operatorname{sign}\left(z_{0}-f(t)\right) \\
& +z_{1} \\
& \dot{z}_{1}=v_{1} \text {, } \\
& v_{1}=-\lambda_{1} L^{2 /(n+1)}\left|z_{1}-v_{0}\right|^{(n-1) / n} \operatorname{sign}\left(z_{1}-v_{0}\right)+z_{2} \text {, } \\
& \vdots \\
& \dot{z}_{n-1}=v_{n-1} \text {, } \\
& v_{n-1}=-\lambda_{n-1} L^{1 / 2}\left|z_{n-1}-v_{n-2}\right|^{1 / 2} \operatorname{sign}\left(z_{n-1}-v_{n-2}\right) \\
& +z_{n} \\
& \dot{z}_{n}=-\lambda_{n} L \operatorname{sign}\left(z_{n}-v_{n-1}\right),
\end{aligned}
$$

and if $\lambda_{i}>0$ is sufficiently large, the convergence is guaranteed.

To obtain the third-order derivative of $\dot{q}$, we construct the third-order sliding mode differentiator and estimate the derivative of $\dot{q}$ for each order. In view of differential precision, we configure the following fifth-order differentiator. See Appendix A for comparison:

$$
\begin{aligned}
& \dot{z}_{0}=v_{0}, \\
& v_{0}=-\lambda_{0} L^{1 / 6}\left|z_{0}-\dot{q}\right|^{5 / 6} \operatorname{sign}\left(z_{0}-\dot{q}\right)+z_{1}, \\
& \dot{z}_{1}=v_{1}, \\
& v_{1}=-\lambda_{1} L^{1 / 5}\left|z_{1}-v_{0}\right|^{4 / 5} \operatorname{sign}\left(z_{1}-v_{0}\right)+z_{2}, \\
& \dot{z}_{2}=v_{2}, \\
& v_{2}=-\lambda_{2} L^{1 / 4}\left|z_{2}-v_{1}\right|^{3 / 4} \operatorname{sign}\left(z_{2}-v_{1}\right)+z_{3}, \\
& \dot{z}_{3}=v_{3}, \\
& v_{3}=-\lambda_{3} L^{1 / 3}\left|z_{3}-v_{2}\right|^{2 / 3} \operatorname{sign}\left(z_{3}-v_{2}\right)+z_{4}, \\
& \dot{z}_{4}=v_{4}, \\
& v_{4}=-\lambda_{4} L^{1 / 2}\left|z_{4}-v_{3}\right|^{1 / 2} \operatorname{sign}\left(z_{4}-v_{3}\right)+z_{5}, \\
& \dot{z}_{5}=v_{5}, \\
& v_{5}=-\lambda_{5} L \operatorname{sign}\left(z_{5}-v_{4}\right),
\end{aligned}
$$

where $z_{3}, z_{2}, z_{1}$, and $z_{0}$ are the estimations of $q^{(4)}, \ddot{q}, \ddot{q}$, and $\dot{q}$.

\section{Baseline Separated Guidance and Control Method}

To verify the homing performance of the integrated guidance and control method, we compare it with the separated guidance and control method. The guidance laws we used are the proportional navigation ( $\mathrm{PN}$ ) guidance law for attacking a nonmaneuvering target and the optimal sliding mode guidance law for attacking a maneuvering target.

4.1. The Proportional Navigation (PN) Guidance Law. The guidance law has a simple formula and excellent performances for nonmaneuvering target. Its formula is as follows:

$$
n_{c}=-N \dot{q} \frac{V_{M}}{g} ;
$$

$n_{c}$ is the overload command; $N$ is the effective navigation ratio; $\dot{q}$ is the LOS rate; $V_{M}$ is the speed of the missile; $g$ is the acceleration of the gravity. The guidance law gives the overload command of the missile according to the LOS rate, and then the controller gives the rudder deflection command according to the overload command.

4.2. The Optimal Sliding Mode Guidance Law. The optimal sliding mode guidance law (OSMG) is a novel practical guidance law proposed by D. Zhou. He combines the optimal guidance law with the sliding mode guidance law and designs the new sliding mode guidance law that not only is robust to maneuvering target but also has the merits of the optimal guidance law such as good dynamic performance and energy conservation. Its formula is as follows:

$$
n_{c}=-3\left|\dot{R}_{0}\right| \dot{q}+\varepsilon \frac{\dot{q}}{|\dot{q}|+\delta},
$$

where $n_{c}$ is the overload command; $\dot{R}_{0}$ is the approach velocity of the missile and its target; $\dot{q}$ is their LOS rate; $\varepsilon=$ const is the compensatory gain; $\dot{q} /(|\dot{q}|+\delta)$ is for substituting for $\operatorname{sign}(\dot{q})$ and for smoothing; $\delta$ is a small quantity which could adjust the chattering.

4.3. Separated Guidance and Control Design. For simulation and comparison, we use the conventional three-loop overload autopilot as the controller, which gives the rudder deflection command according to the feedback of the three loops of overload, pseudo-angle of attack, and pitch rate. The block diagram is as shown in Figure 3.

As the figure shows, the inner loop has the feedback on angular velocity, which improves the damping characteristics of the missile airframe.

According to the aerodynamic coefficient of the missile with selected working points, we set $K_{I}=0.19, K_{\alpha}=3$, and $K_{\omega}=-0.25$ and the controller can well track the overload command; the rise time of its step response is 0.46 seconds, and its settling time is 0.83 seconds. The step responses of the missile to overload command and the Bode diagram for open loop are shown in Figure 4. 


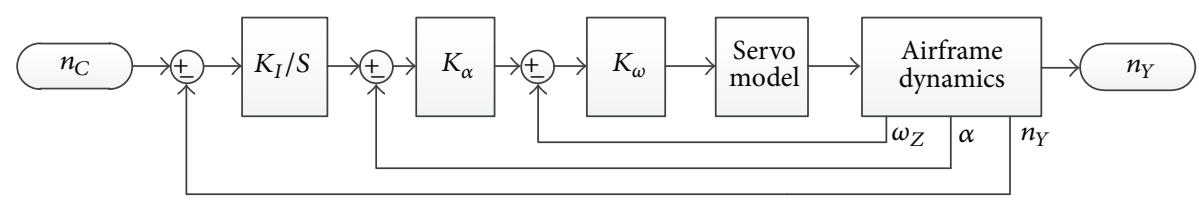

FIGURE 3: The working principles for three-loop overload autopilot.
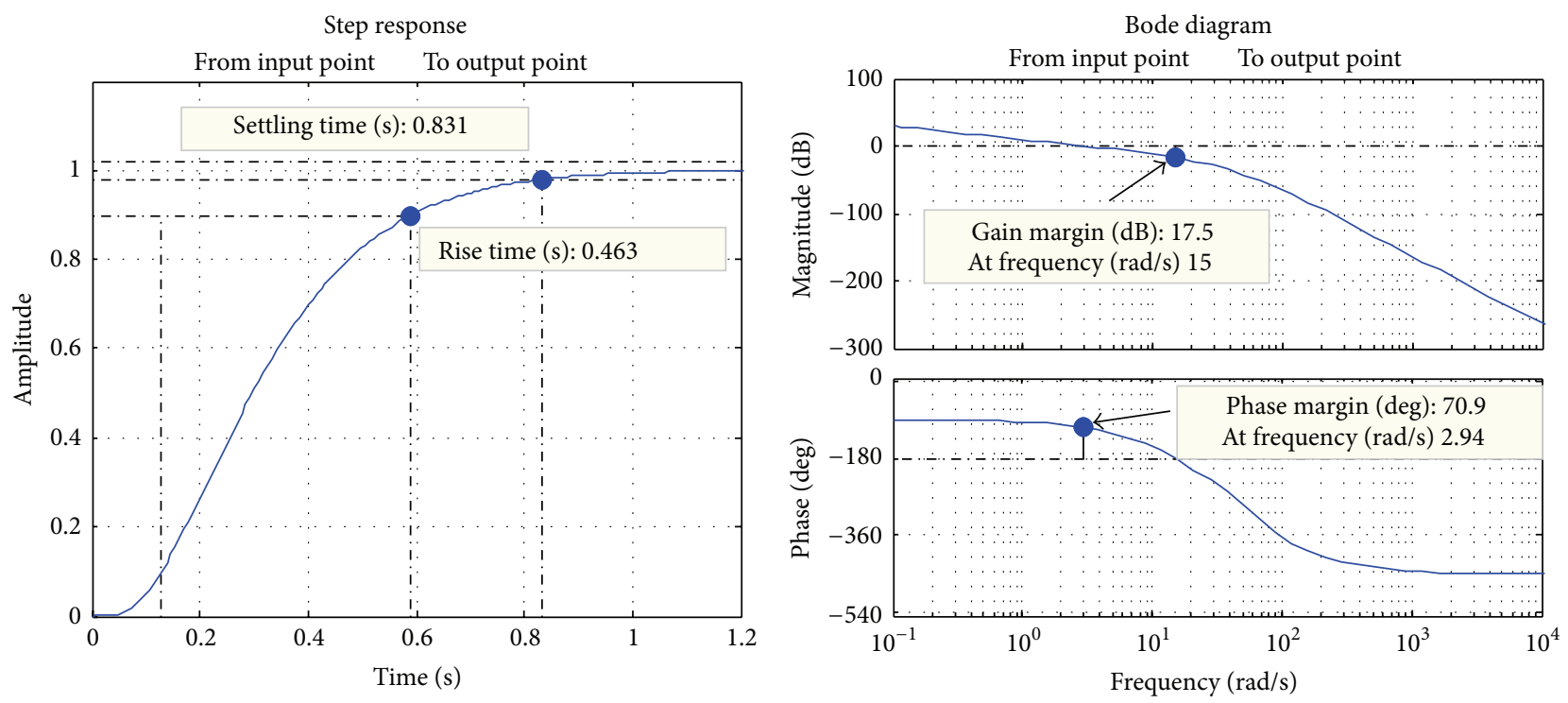

FIgURE 4: The autopilot performance: step response and Bode diagram.

\section{Simulation Results}

To verify the high order sliding mode integrated guidance and control (HOSM-IGC) method, we compare it with the baseline separated guidance and control. Numerical simulations are designed in three typical engagement scenarios.

AORED parameters are as follows: the initial value $\lambda_{0}=$ $\lambda_{1}=\lambda_{2}=\lambda_{3}=50 ; z_{0}=0.1 ; z_{1}=z_{2}=z_{3}=0 ; L=1400$; the simulation step is 0.0001 seconds.

5.1. Scenario 1: Nonmaneuvering Target. In the first scenario, the nonmaneuvering target does uniform rectilinear motion and $\mathrm{PN}$ guidance law with three-loop autopilot is introduced for a comparison with the HOSM-IGC. The initial conditions are set as shown in Table 1 .

The motion equations of the target are as follows:

$$
\begin{aligned}
& \dot{y}_{T}=V_{T} \cos \theta_{T}, \\
& \dot{x}_{T}=-V_{T} \sin \theta_{T}, \\
& \dot{\theta}_{T}=\frac{a_{T}}{V_{T}},
\end{aligned}
$$

$$
a_{T}=0
$$

The simulation results are shown in Figures 5 and 6.

The missile's flight trajectory and overload curve show that, within the first $3 \mathrm{sec}$, the HOSM-IGC method spends much energy (overload) on changing the initial LOS direction
TABLE 1: Initial conditions.

\begin{tabular}{lcc}
\hline Variable & Description & Value \\
\hline$\left(X_{M}, Y_{M}\right)$ & Missile initial position & $(0 \mathrm{~km}, 18 \mathrm{~km})$ \\
$\left(X_{T}, Y_{T}\right)$ & Target initial position & $(60 \mathrm{~km}, 20 \mathrm{~km})$ \\
$V_{M}$ & Missile initial velocity & $1200(\mathrm{~m} / \mathrm{s})$ \\
$V_{T}$ & Target initial velocity & $2000(\mathrm{~m} / \mathrm{s})$ \\
$\theta_{M}$ & Missile initial flight path angle & $0(\mathrm{deg})$ \\
$\theta_{T}$ & Target initial flight path angle & $0(\mathrm{deg})$ \\
$\beta$ & Parameter of the controller & 10 or 30 \\
$N$ & Navigation ratio of PN & 3 \\
\hline
\end{tabular}

of the missile. After the boresight adjustment, $\sigma$ reaches the desired sliding manifold, and the missile does not need any maneuver to hit the target. This is because the target does not maneuver any more, which means no disturbance is introduced in the engagement dynamic, so the state (or the sliding mode) of the missile will stay on the manifold. In contrast, the overload command given by the PN guidance law increases fast as the relative distance decreases. To examine the performances of the two guidance laws further, we increase the target speed and analyze the command changes.

Therefore, we set the target speeds, $3000 \mathrm{~m} / \mathrm{s}, 4000 \mathrm{~m} / \mathrm{s}$, and carry out simulations. The simulation result is shown in the overload curve in Figure 7. 


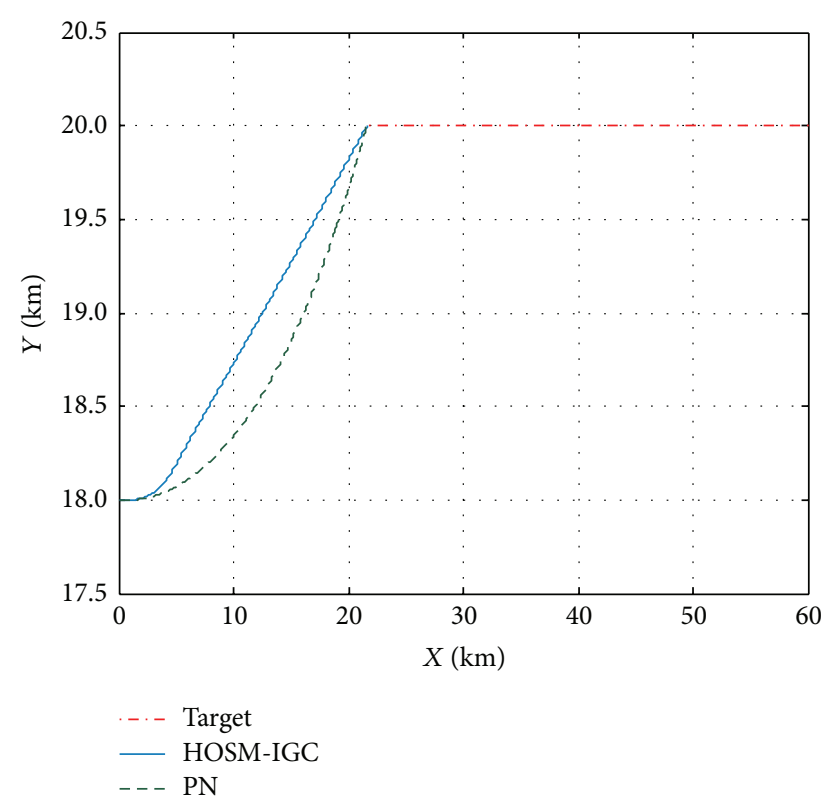

Figure 5: Target and missile trajectories.

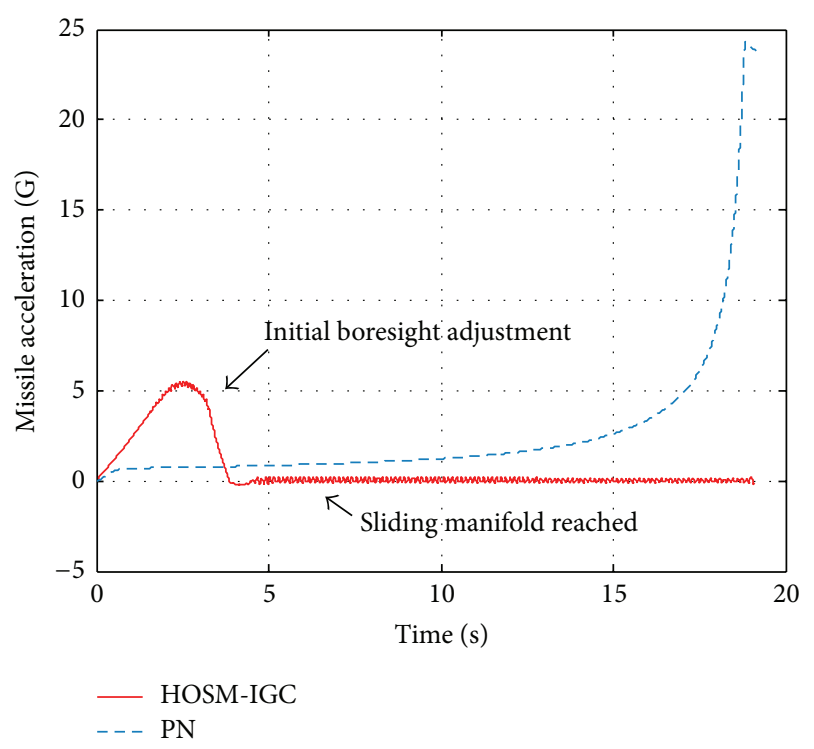

FIgure 6: Missile acceleration profile.

It is evident that the speed of divergence of the overload command given by the PN guidance law increases with the target speed. Specifically, when the target speed reaches $4000 \mathrm{~m} / \mathrm{s}$, the commanded overload is almost $30 \mathrm{~g}$ which is obviously not ideal for the attack of a nonmaneuvering target, but, with the HOSM-IGC method, the missile adjusts its boresight very quickly, then maintains it around $0 \mathrm{~g}$, and flies to its target in the rectilinear ballistic trajectory, not affected by the increases of target speed, still accomplishing the highprecision hit-on collision.

Figure 8 shows that although the HOSM-IGC method achieves a more effective overload command, on the other side, it sees some chattering when the sliding mode reaches

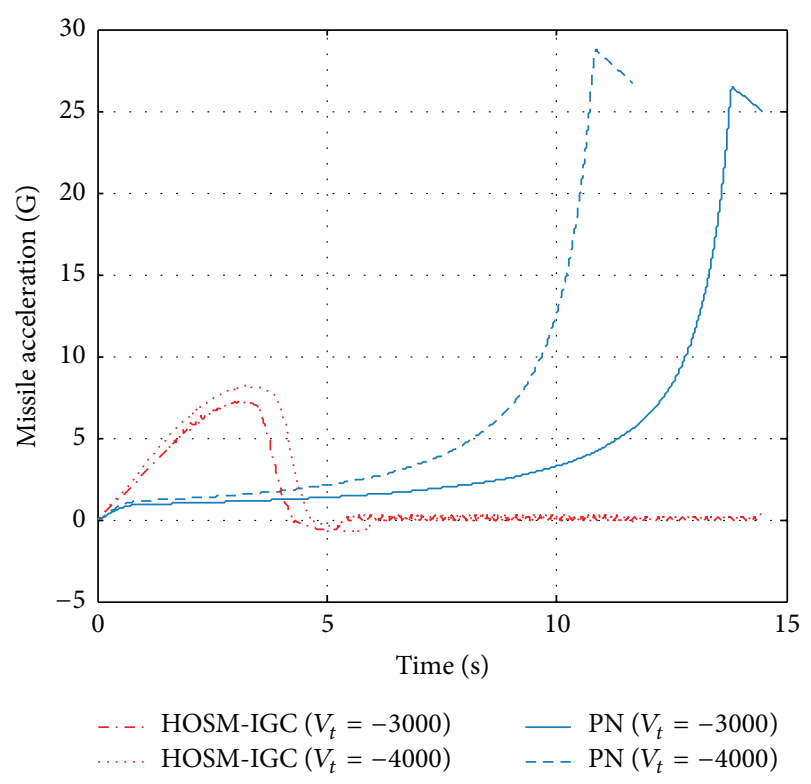

FIGURE 7: Missile acceleration profile.

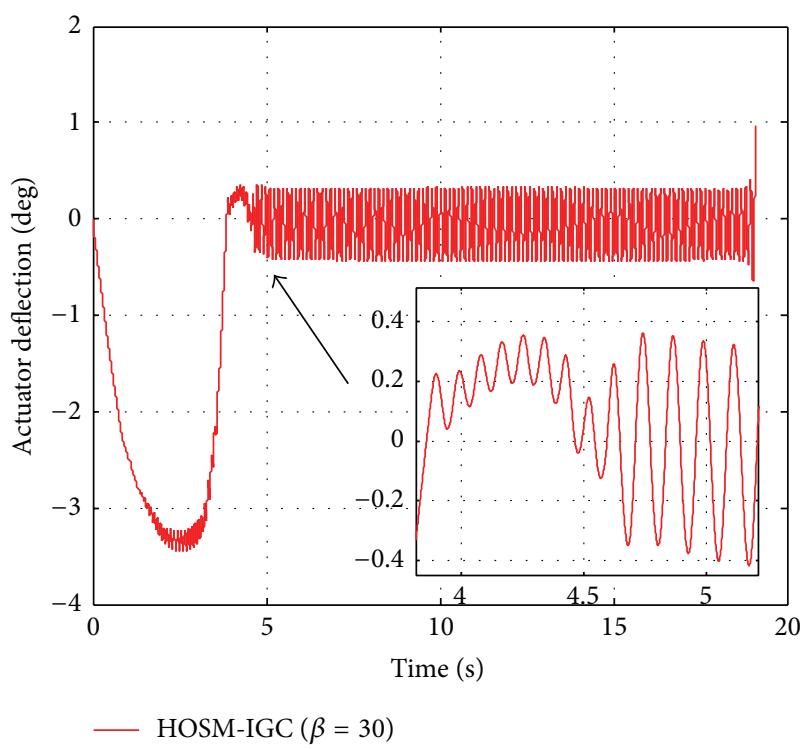

FIgURE 8: Actuator deflection.

the desired manifold; the commanded rudder deflection angle chatters for around $0.45 \mathrm{deg}$ at about $15 \mathrm{~Hz}$ which is kind of harmful to the system. To reduce the chattering, we adjust the controller's parameter $\beta=10$ and carry out simulations again. Figure 9 shows that after $\beta$ decreases, the command of rudder deflection angle converges slower (for about $7 \mathrm{sec}$ ); however, the chattering weakens obviously, its magnitude being only about 0.15 degrees. The smaller chattering well enhances convergence precision, eventually reducing the target missing. The reason that minor $\beta$ results in an alleviative chattering can be seen from (47). Figure 10 shows the target and missile trajectories.

Table 2 compares the average miss distance of 50 simulations under the conditions discussed above; the comparison 
TABLE 2: Average miss distance of 50 simulations.

\begin{tabular}{lccc}
\hline Target speed & PN & $\begin{array}{c}\text { HOSM-IGC } \\
(\beta=30)\end{array}$ & $\begin{array}{c}\text { HOSM-IGC } \\
(\beta=10)\end{array}$ \\
\hline$V_{t}=2000 \mathrm{~m} / \mathrm{s}$ & $1.15 \mathrm{~m}$ & 0.86 & $0.73 \mathrm{~m}$ \\
$V_{t}=3000 \mathrm{~m} / \mathrm{s}$ & $2.61 \mathrm{~m}$ & 1.17 & 1.06 \\
$V_{t}=4000 \mathrm{~m} / \mathrm{s}$ & $5.42 \mathrm{~m}$ & 1.56 & 1.34 \\
\hline
\end{tabular}

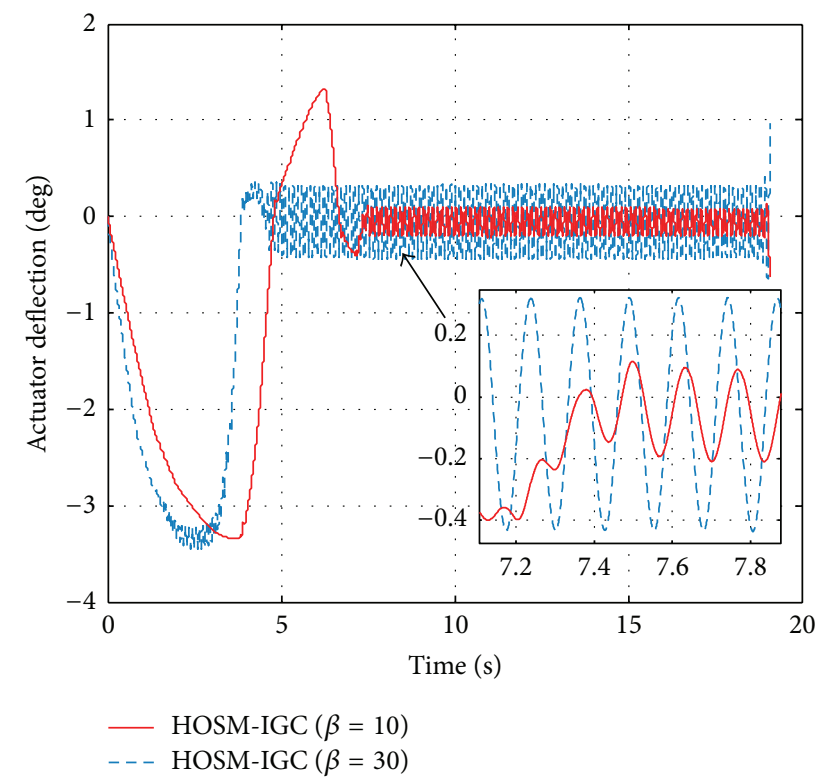

Figure 9: Actuator deflection.

results show that when the missile intercepts a nonmaneuvering target, the hit precision of the HOSM-IGC method is apparently higher than that of the PN guidance law and that the low-gain HOSM-IGC method can effectively reduce the chattering magnitude, thus enhancing the interception precision.

5.2. Scenario 2: Step Maneuvering Target. At the first stage, the target flies at uniform speed and in a rectilinear way; after 10 seconds, it maneuvers at the normal acceleration of $5 \mathrm{~g}$. In this scenario, OSMG is introduced for a comparison with the HOSM-IGC:

$$
\begin{aligned}
& \dot{y}_{T}=V_{T} \cos \theta_{T}, \\
& \dot{x}_{T}=-V_{T} \sin \theta_{T}, \\
& \dot{\theta}_{T}=\frac{a_{T}}{V_{T}},
\end{aligned}
$$

$$
a_{T}= \begin{cases}0 \mathrm{~g}, & t<10 \mathrm{sec} \\ 5 \mathrm{~g}, & t>10 \mathrm{sec}\end{cases}
$$

The initial simulation conditions are given in Table 3.

The overload curve in Figure 11 shows dearly that both types of guidance laws can track the maneuvering target. During 0 to 10 seconds, the target flies at uniform speed and in a rectilinear way, and the missile converges its overload to
TABLE 3: Initial conditions.

\begin{tabular}{lcc}
\hline Variable & Description & Value \\
\hline$\left(X_{M}, Y_{M}\right)$ & Missile initial position & $(0 \mathrm{~km}, 22 \mathrm{~km})$ \\
$\left(X_{T}, Y_{T}\right)$ & Target initial position & $(60 \mathrm{~km}, 20 \mathrm{~km})$ \\
$V_{M}$ & Missile initial velocity & $1200(\mathrm{~m} / \mathrm{s})$ \\
$V_{T}$ & Target initial velocity & $2000(\mathrm{~m} / \mathrm{s})$ \\
$\theta_{M}$ & Missile initial flight path angle & $0(\mathrm{deg})$ \\
$\theta_{T}$ & Target initial flight path angle & $0(\mathrm{deg})$ \\
$\varepsilon$ & Compensatory gain & 3 \\
$\delta$ & Saturation parameter & 0.001 \\
\hline
\end{tabular}

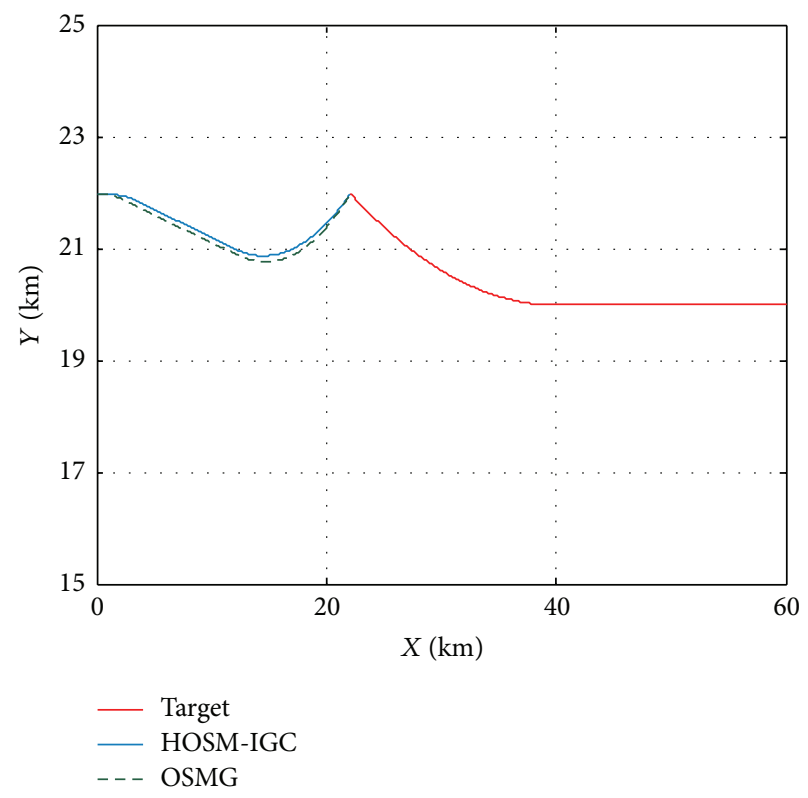

Figure 10: Target and missile trajectories.

$0 \mathrm{~g}$ and flies to its target; after 10 seconds, the target begins to maneuver by $5 \mathrm{~g}$. Both types of guidance law respond by rapidly increasing the overload, adjusting attitude, and making the missile's boresight aim at its target.

We can see that when both types of guidance law track their targets, the convergence speed of OSMG is almost the same as the HOSM-IGC method. But the HOSM-IGC method has higher convergence precision and needs lower overload at the end phase.

We can also see that, after the target maneuvers, if the missile is given enough time to track the target's maneuver, namely, let the missile's overload command converge to the overload of the target, there may not be large miss distance. In other words, for a certain period of time before the collision, the target maneuver (it means only a limited maneuver which does not include the condition that the maneuvering of the target for a long time may cause a change of the geometrical relations between the missile and its target) has a small effect on both types of guidance law.

But if the maneuver occurs rather late, namely, within one to three seconds before collision, when the overload command of guidance law is not yet converged, the approaching 


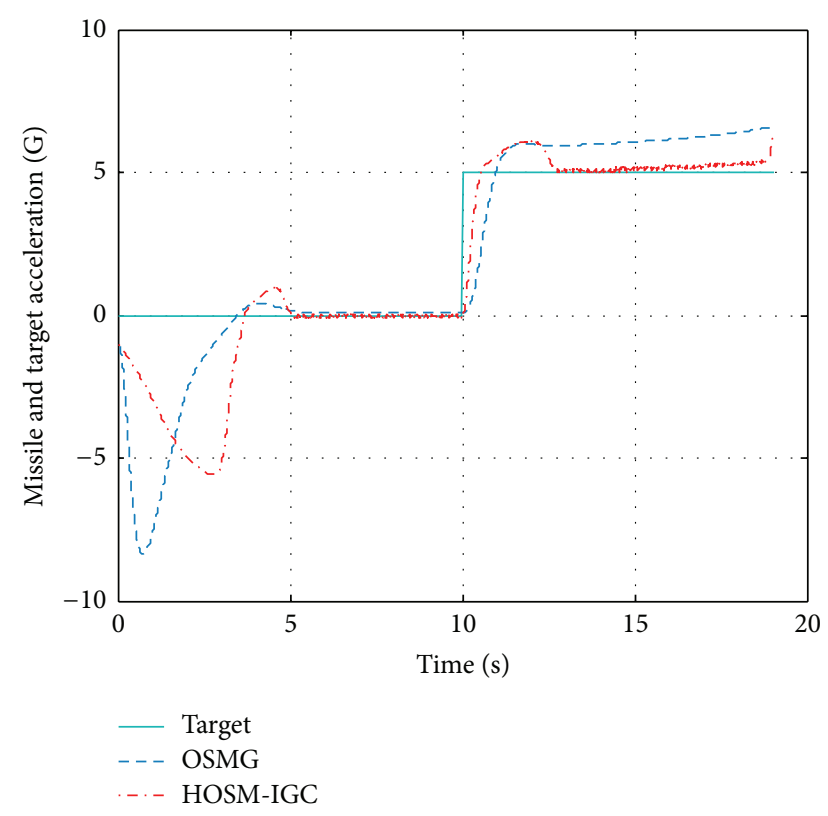

FIgUre 11: Missile and target acceleration profile.

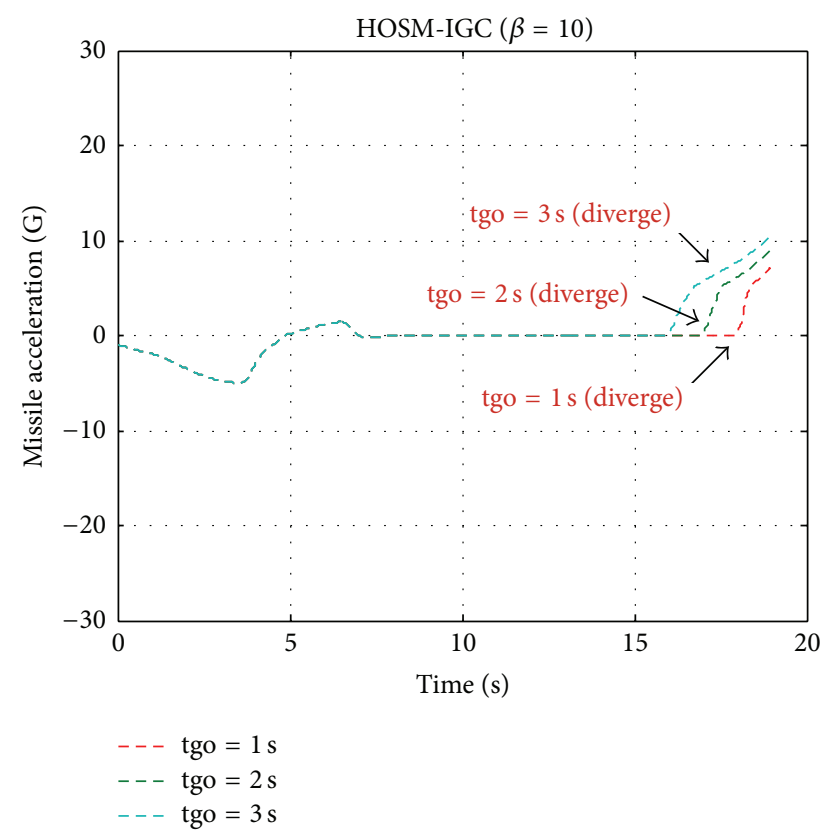

Figure 12: Missile acceleration profile.

collision increases the miss distance. Therefore, increasing the parameter $\beta$ can remarkably increase the convergence speed and effectively enhance guidance precision. As Figure 12 shows, when $\beta=10$, the overload at the end phase converges slowly, even if the target maneuvers three seconds before collision; the missile's overload still has no time to converge, being unable to track the maneuvering target. Figure 13 shows that when $\beta$ increases to 30 and tgo $=3$ seconds, the overload can converge to about $5 \mathrm{~g}$, but when tgo $=2 \mathrm{sec}-$ onds, the overload may continue to increase, indicating that

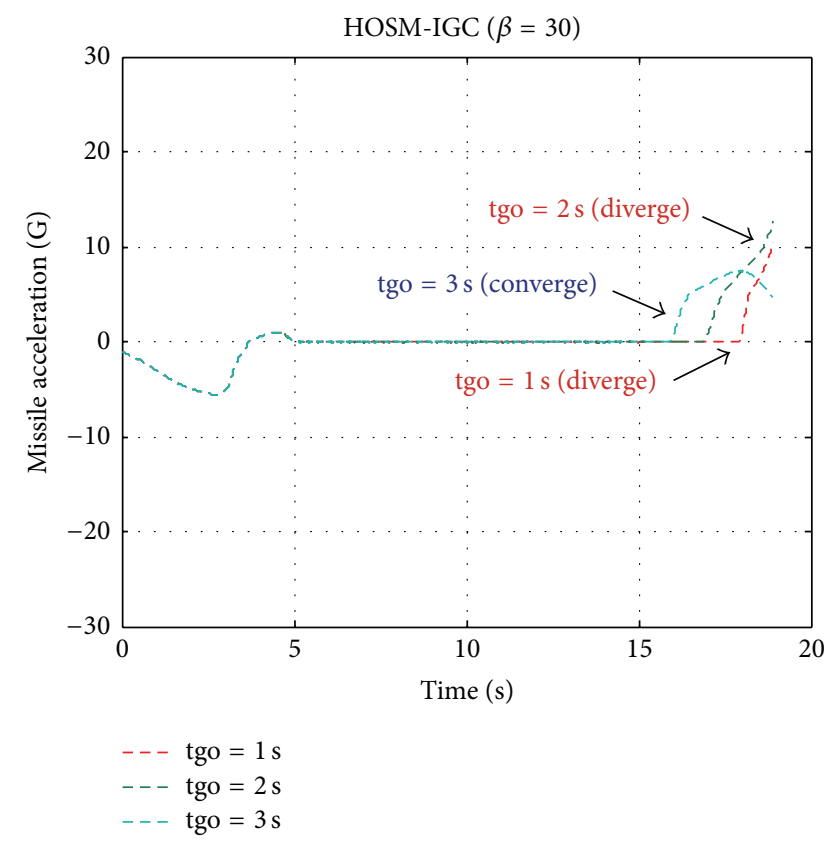

Figure 13: Missile acceleration profile.

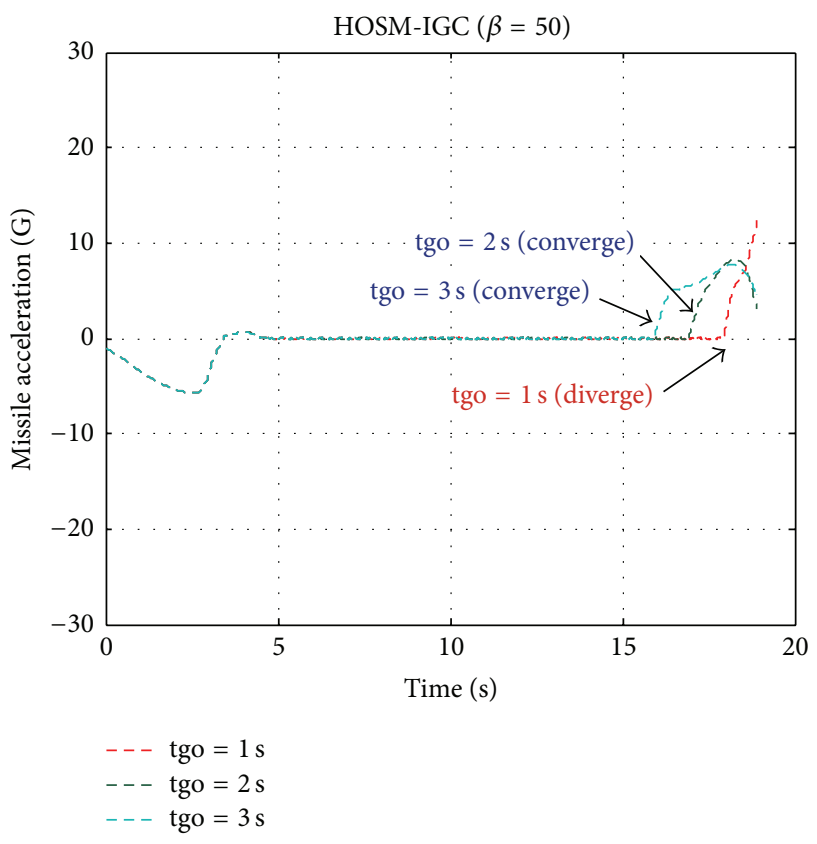

FIGURE 14: Missile acceleration profile.

the convergence still needs to be quickened, thus continuing to rise $\beta$ to 50 . Figure 14 shows that the HOSM-IGC method can track the target that maneuvers when tgo $=2$ seconds; but it causes the divergence of overload and the increase of miss distance if the target maneuvers when tgo $=1$ second. Figure 15 gives the overload curve of the OSMG guidance law and shows that the maneuver of the target before collision may cause the large-scale oscillation of the missile's overload, which may diverge to a large numerical value when the collision occurs in the end. 
TABLE 4: Average miss distances of 50 simulations.

\begin{tabular}{lcccc}
\hline $\begin{array}{l}\text { Target } \\
\text { maneuvering } \\
\text { timing }\end{array}$ & $\begin{array}{c}\text { HOSM-IGC } \\
\beta=30\end{array}$ & $\begin{array}{c}\text { HOSM-IGC } \\
\beta=40\end{array}$ & $\begin{array}{c}\text { HOSM-IGC } \\
\beta=50\end{array}$ & OSMG \\
\hline $\begin{array}{l}\text { tgo }=1 \\
\text { second }\end{array}$ & 4.3539 & 3.224 & 2.8936 & 3.6116 \\
$\begin{array}{l}\text { tgo }=2 \\
\text { seconds }\end{array}$ & 2.5424 & 1.8665 & 0.9322 & 3.5534 \\
tgo $=3$ & 0.8124 & 0.8265 & 0.7538 & 1.1959 \\
seconds & & & & \\
\hline
\end{tabular}

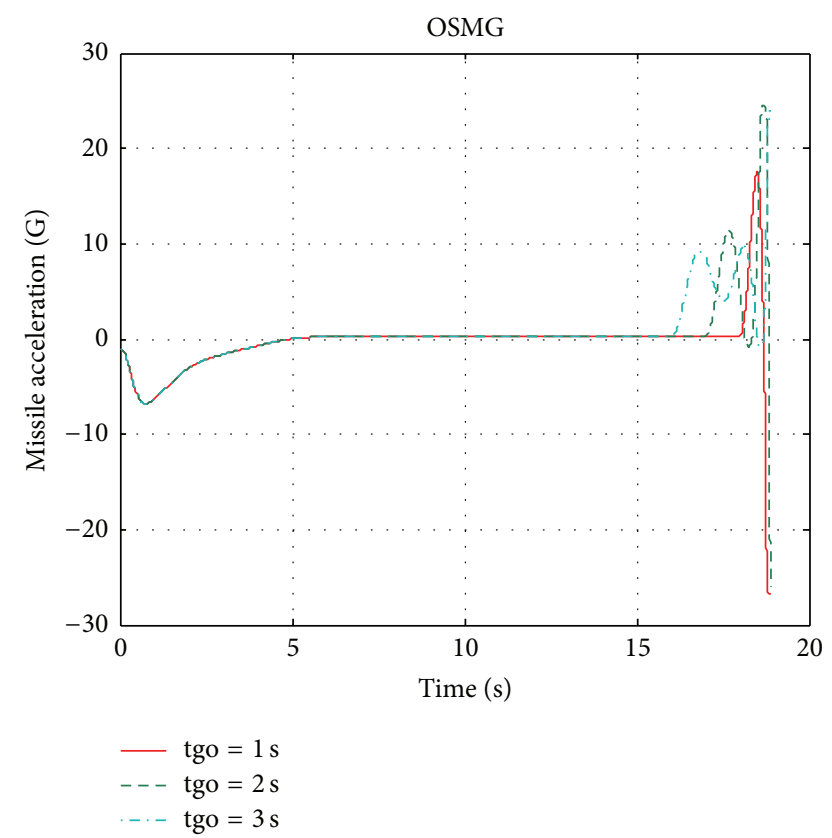

FIGURE 15: Missile acceleration profile.

The analysis in Figures 12, 13, 14, and 15 shows that the overload of the missile converges faster and its miss distance is smaller with increasing $\beta$. To verify this finding, we carry out 50 times Monte Carlo simulations, in which the position and speed of the target have $1 \%$ of random difference.

The average miss distances are shown in Table 4 and Figure 16. Clearly, the timing of the target's step maneuver dramatically affects the final interception precision, more specifically; given a shorter reaction time for the guidance and control system, the missile seems more likely to miss the target. To the OSMG guidance law, in all three scenarios, hardly does it show any advantages against the HOSM-IGC. It can also be seen that, with the increasing of the $\beta$, the HOSMIGC system responds even faster which leads to an obvious decrease of the average miss distance. The effect of $\beta$ on the response of the HOSM-IGC system is a valuable guideline when implementing the proposed method into practice.

5.3. Scenario 3: Weaving Target. In this scenario, the target maneuvers by $a_{T}=40 \sin (\pi t / 2)$. OSMG with three-loop
TABLE 5: Initial conditions.

\begin{tabular}{lcc}
\hline Variable & Description & Value \\
\hline$\left(X_{M}, Y_{M}\right)$ & Missile initial position & $(0 \mathrm{~km}, 17.5 \mathrm{~km})$ \\
$\left(X_{T}, Y_{T}\right)$ & Target initial position & $(60 \mathrm{~km}, 19.5 \mathrm{~km})$ \\
$V_{M}$ & Missile initial velocity & $1200(\mathrm{~m} / \mathrm{s})$ \\
$V_{T}$ & Target initial velocity & $2000(\mathrm{~m} / \mathrm{s})$ \\
$\theta_{M}$ & Missile initial flight path angle & $0(\mathrm{deg})$ \\
$\theta_{T}$ & Target initial flight path angle & $0(\mathrm{deg})$ \\
$\varepsilon$ & Compensatory gain & 3 \\
$\delta$ & Saturation parameter & 0.001 \\
\hline
\end{tabular}

autopilot is introduced for comparison. The motion equations of the target are as follows:

$$
\begin{aligned}
& \dot{y}_{T}=V_{T} \cos \theta_{T}, \\
& \dot{x}_{T}=-V_{T} \sin \theta_{T}, \\
& \dot{\theta}_{T}=\frac{a_{T}}{V_{T}},
\end{aligned}
$$

$$
a_{T}=40 \sin \left(\frac{\pi t}{2}\right) .
$$

The initial simulation conditions are given in Table 5.

The missile's trajectory under the two guidance and control methods given in Figure 17 does not see much difference.

However, the overload curve given in Figure 18 shows that after the missile completes its initial attitude adjustment, with the HOSM-IGC method, it can almost ideally track its maneuvering target; by contrast, with the OSMG method, the missile seems to have the tendency to track its target's maneuver but has larger tracking errors. Besides, with the OSMG method, the missile's overload increases rapidly at the end of attack primarily because of the divergence of its LOS rate. On the other hand, with the HOSM-IGC method, the missile has no divergence even at the end of attack, ensuring a smaller target missing quantity.

The actuator deflection curve in Figure 19 shows that, in order to provide a rather big normal overload for the end phase, the OSMG method produces a rather big rudder deflection command; however, it may increase the missile's target missing quantity once its rudder deflection saturates and the missile does not have enough overloads, or the rudder cannot respond that fast.

As shown in Figure 20, because of the dramatic change in overload command, the response of the missile's autopilot to high-frequency command sees an obvious phase lag and amplitude value attenuation; its actual overload cannot track the command ideally; this is a main reason why the miss distance increases. However, the controller in the HOSMIGC method gives its rudder deflection command directly, and there is no lagging or attenuation caused by the autopilot, thus enhancing the guidance precision effectively. Furthermore, the fast convergence of the high order sliding mode makes the missile rapidly track its maneuvering target with the most reasonable rudder deflection command, reducing its overload effectively. 

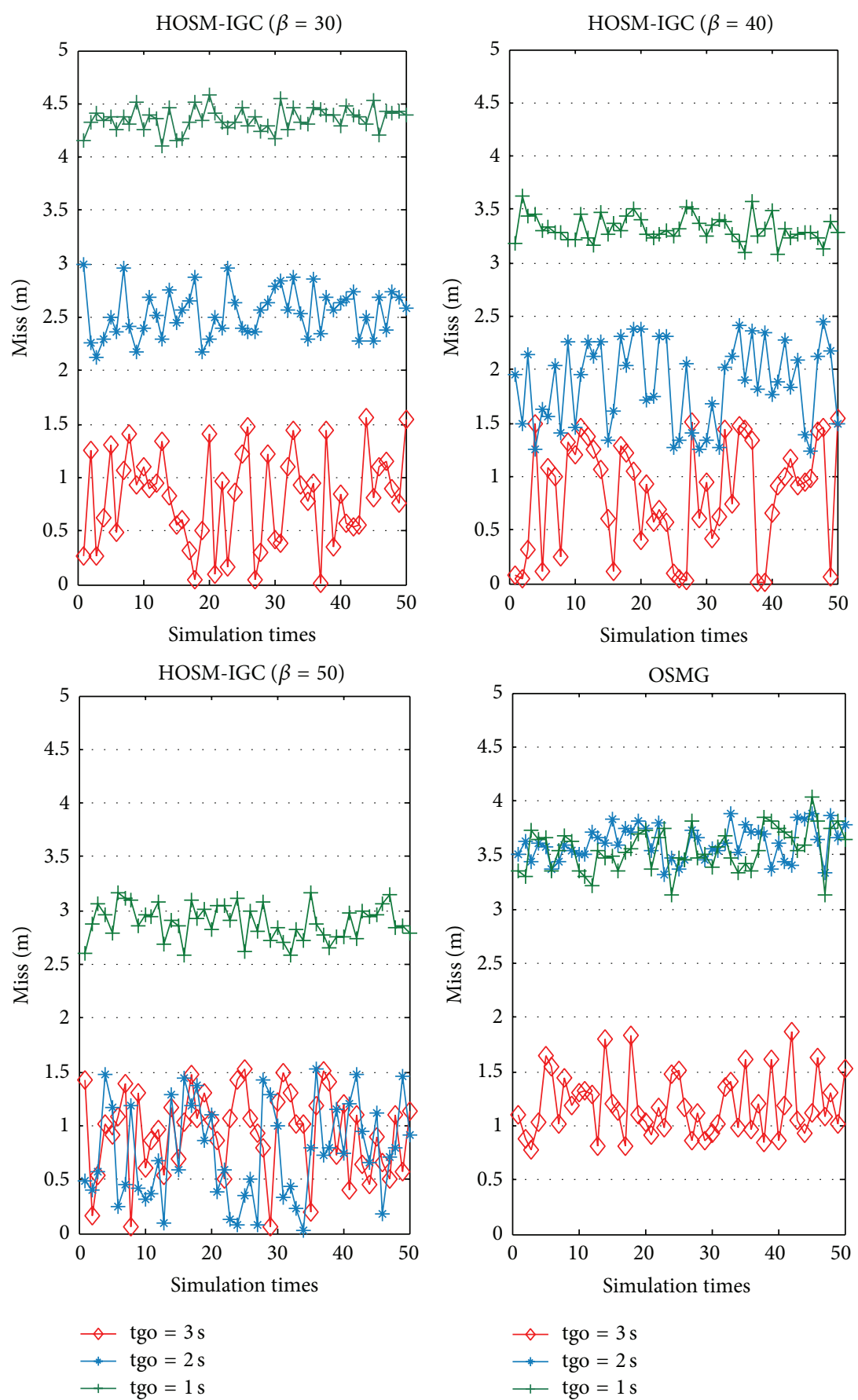

FIGURE 16: Miss distances of HOSM-IGC $(\beta=30,40,50)$ and OSMG with target maneuvers at different time-to-go (tgo $=1$ second, 2 seconds, and 3 seconds).

The average miss distance of 50 simulations under the conditions is $0.73 \mathrm{~m}$ for HOSM-IGC and $1.86 \mathrm{~m}$ for OSMG. We can see that the HOSM-IGC method not only does provide a more reasonable actuator deflection command, but also achieves a higher interception precision.

\section{Conclusions}

This paper proposes an LOS feedback integrated guidance and control method using quasi-continuous high order sliding mode guidance and control method. With the fast and precise convergence of the quasi-continuous HOSM method, the HOSM-IGC method performs much better than the traditional separated guidance and control method with less acceleration effort and less miss distance in all the three simulation scenarios of nonmaneuvering target, step maneuvering target, and weaving target. In addition, the idea of virtual control largely alleviates the chattering without any sacrifice of robustness. As a result of the alleviation of the chattering, the control input command $\delta_{Z}$ becomes 


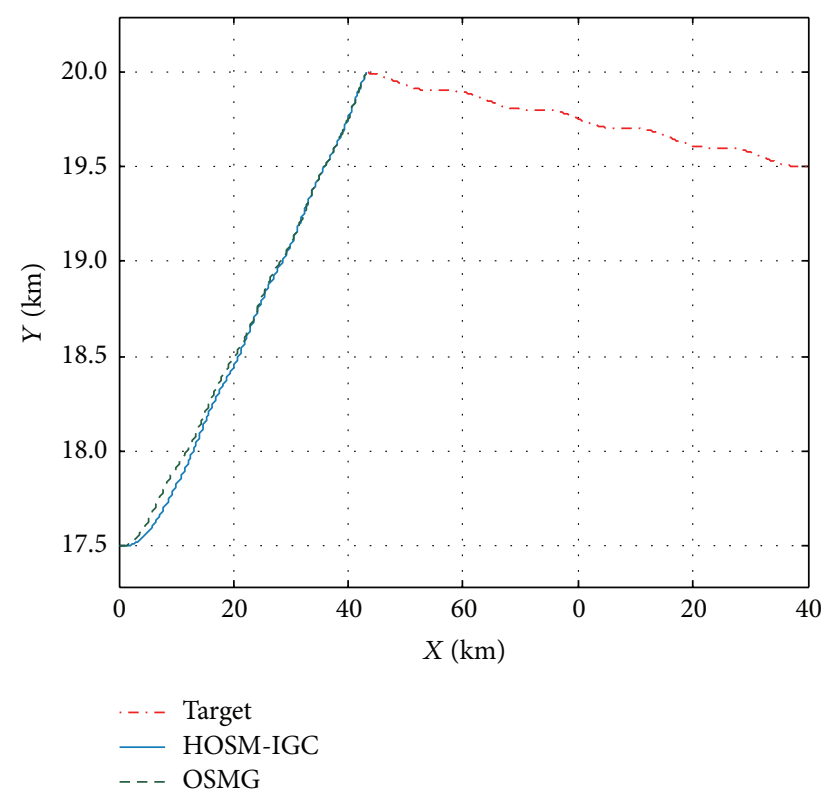

Figure 17: The trajectories of the missile and its target.

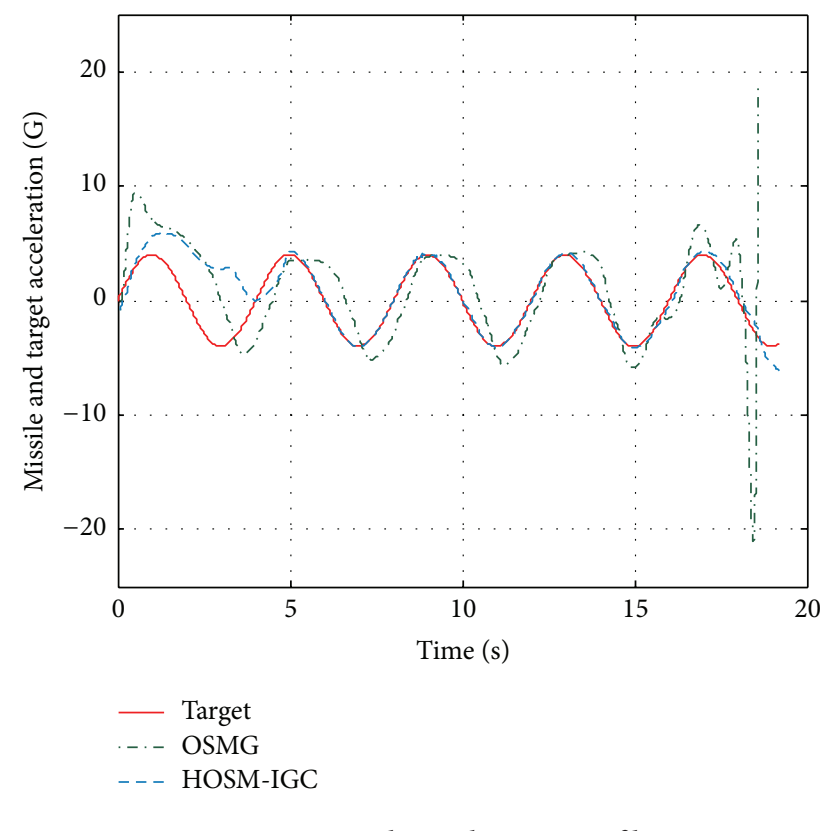

FIGURE 18: Missile acceleration profile.

smooth enough for the actuator to be implemented; as a result the interception accuracy is improved. In designing the quasi-continuous sliding mode controller, the ArbitraryOrder Robust Exact Differentiator is introduced to estimate the high order time derivative of the LOS rate $\dot{q}$, which not only ensures the guidance and control precision but also simplifies the calculation.

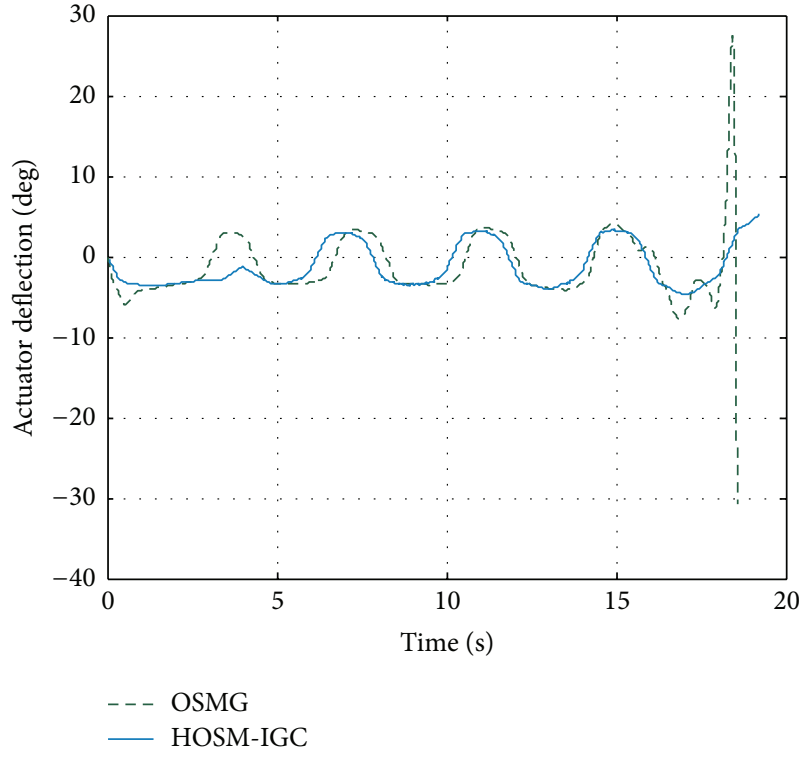

FIgURE 19: Actuator deflection.

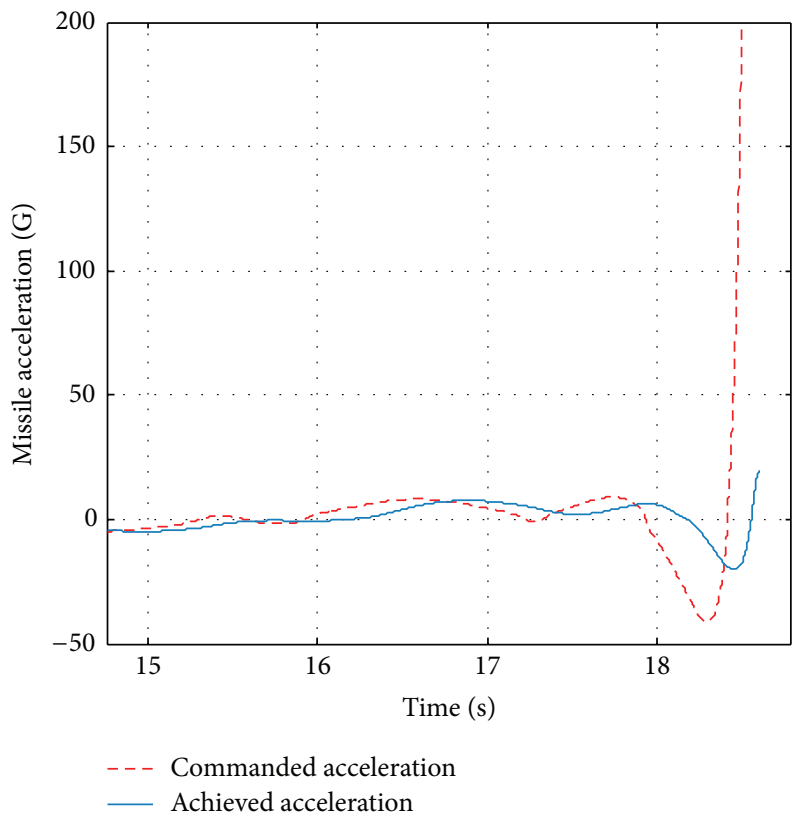

FIgURE 20: Commanded acceleration and achieved acceleration.

\section{Appendices}

\section{A. The Third-Order Robust Exact Differentiator}

The third-order robust exact differentiator built according to the following parameters and equations is used to perform the simulation; the initial value $\lambda_{0}=\lambda_{1}=\lambda_{2}=\lambda_{3}=50$; 

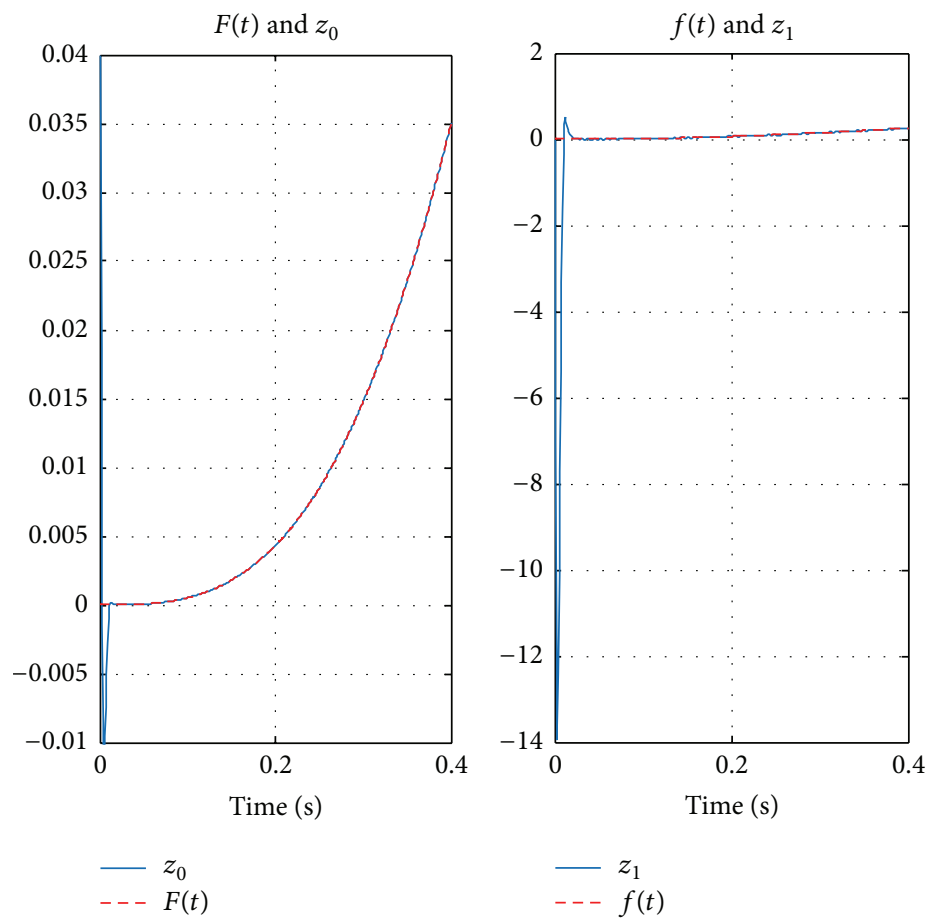

- - $F(t)$
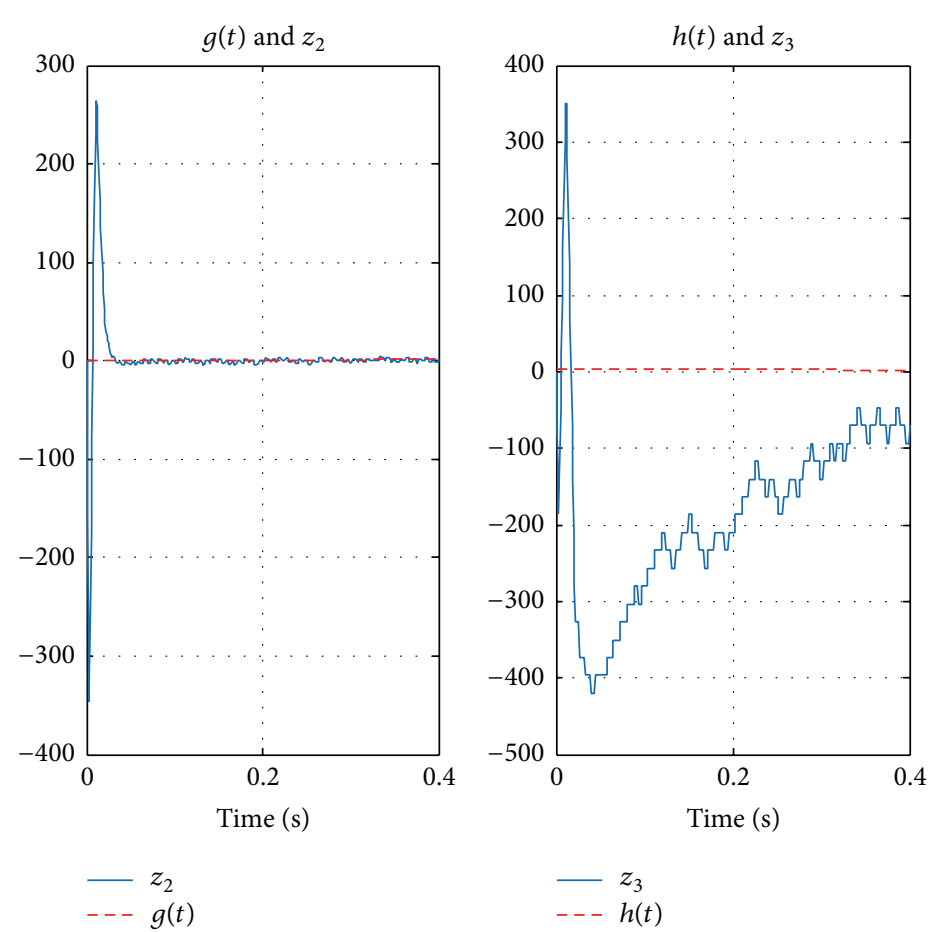

FIgURE 21: The outputs of the differentiator in the sampling interval of 0.001 seconds.

the initial values $z_{0}=0.1, z_{1}=z_{2}=z_{3}=0 ; L=1400$; the simulation step is 0.001 seconds. Consider

$$
\begin{aligned}
& \dot{z}_{0}=v_{0}, \\
& v_{0}=-\lambda_{0} L^{1 / 4}\left|z_{0}-\dot{q}\right|^{3 / 4} \operatorname{sign}\left(z_{0}-\dot{q}\right)+z_{1}, \\
& \dot{z}_{1}=v_{1},
\end{aligned}
$$

$$
\begin{aligned}
& v_{1}=-\lambda_{1} L^{1 / 3}\left|z_{1}-v_{0}\right|^{2 / 3} \operatorname{sign}\left(z_{1}-v_{0}\right)+z_{2}, \\
& \dot{z}_{2}=v_{2}, \\
& v_{2}=-\lambda_{2} L^{1 / 2}\left|z_{2}-v_{1}\right|^{1 / 2} \operatorname{sign}\left(z_{2}-v_{1}\right)+z_{3}, \\
& \dot{z}_{3}=-\lambda_{3} L \operatorname{sign}\left(z_{3}-v_{2}\right) .
\end{aligned}
$$



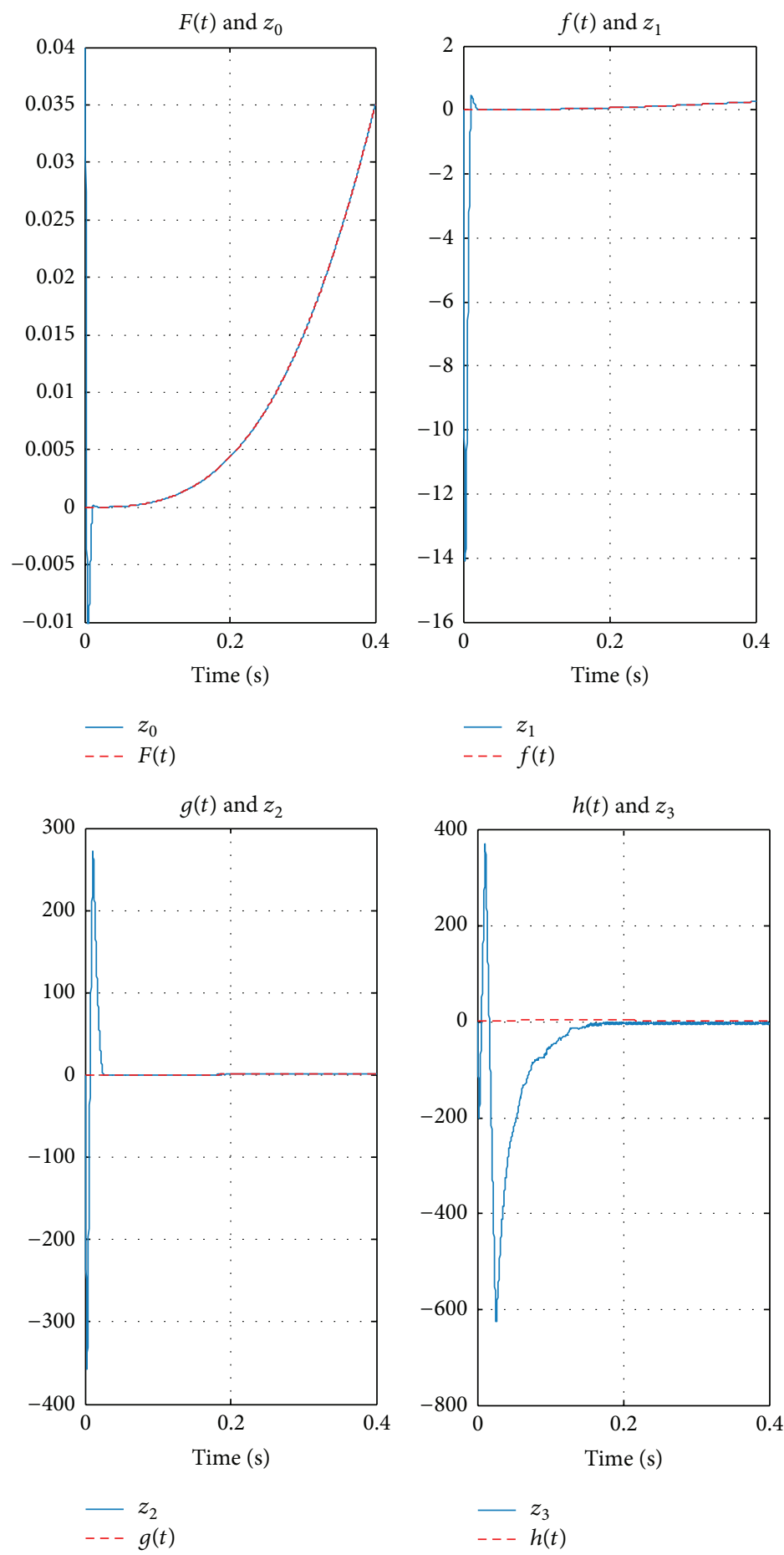

FIGURE 22: The outputs of the differentiator in the sampling interval of 0.0001 seconds.

If the designed signals are $F(t)=\int f(t) d t, f(t)=$ $\int g(t) d t, g(t)=\int h(t) d t$, and $h(t)=\sin (2 \pi t)+$ $3 \cos (\pi t)$, then theoretically $f(t)$ is the first-order differential of input signal; $g(t)$ is the second-order differential of input signal; $h(t)$ is the third-order differential of input signal. Through the simulation, the differential signal of actual output is compared with that of theoretical output; the comparison results are shown in Figure 21.

Figure 21 shows that $z_{0}$ can effectively track the signal $F(t)$, but $z_{2}$ has obvious errors in tracking the second-order differential $g(t) ; z_{3}$ has greater errors in tracking the thirdorder differential; thus the overall differential effect is not ideal. 

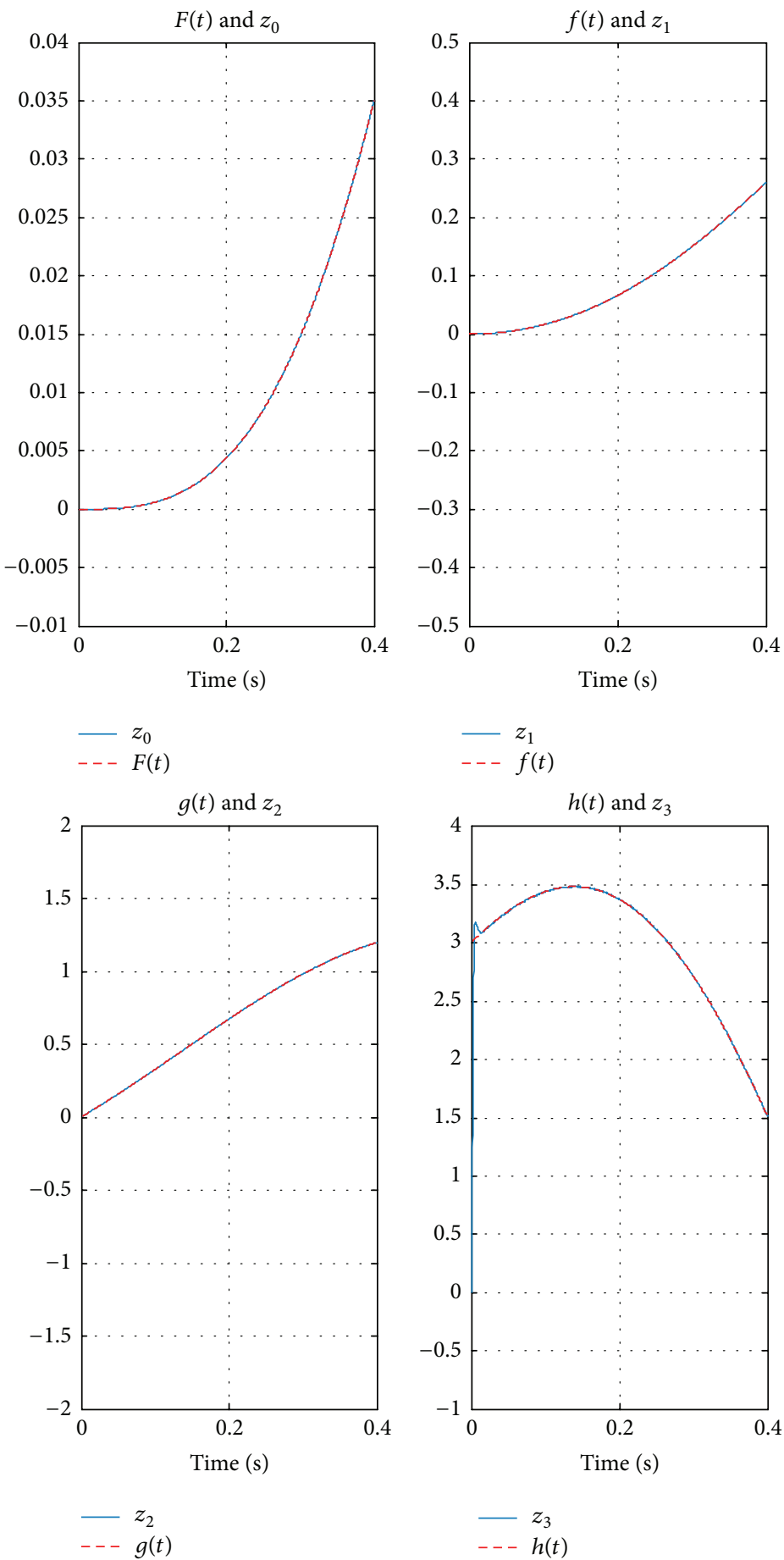

FIGURE 23: The outputs of the differentiator in the sampling interval of 0.0001 seconds.

There are two causes for the errors in a usual differentiator: the setting of sampling interval and the setting of number of orders of the differentiator. The following theorem applies here.

Theorem A.1 (see [19]). If the sampling interval $\tau>0$, the accuracy of the controller is expressed as follows:

$$
\left|\sigma^{(i)}\right| \leq \mu \tau^{r-i}, \quad i=0, \ldots, r-1 .
$$

That is to say, the precision (understood as the differential precision of a differentiator) of the ith order control is in direct proportion to the power of $r-i$ times of sampling interval $\tau$. Therefore, the decrease of iteration step can enhance the differential tracking precision.

Under the conditions that the sampling interval is 10 times smaller and that the setting is 0.0001 seconds, the simulation results are shown in Figure 22. 
TABLE 6: Tracking errors of the AORED with different orders and sampling interval.

\begin{tabular}{lccc}
\hline Orders of AORED & \multicolumn{2}{c}{ Third order } & Fifth order \\
\hline Sample period & $\tau=0.001$ & $\tau=0.0001$ & $\tau=0.0001$ \\
First-order differential error & 0.01 & Negligible & Negligible \\
Second-order differential error & 1.5 & 0.02 & Negligible \\
Third-order differential error & 70 & 2.5 & 0.0005 \\
\hline
\end{tabular}

As Figure 22 shows, the signal tracking is rather ideal; except a peak in the beginning, the tracking error in the firstorder differential tracking can be neglected; $z_{2}$ has an obviously smaller tracking error in the second-order differential $g(t)$ tracking; in the beginning, $z_{3}$ has a rather sharp peak in the third-order differential tracking but converges to a relatively high precision after 0.1 seconds. As we can see, the differential tracking errors for each number of orders are greatly reduced, but the third-order differential tracking error is still rather big. In the following, we will reduce the tracking errors by increasing the number of orders of the differentiator.

\section{B. The Fifth-Order Robust Exact Differentiator}

The settings of the fifth-order differentiator are given as follows:

$$
\begin{aligned}
& \dot{z}_{0}=v_{0}, \\
& v_{0}=-\lambda_{0} L^{1 / 6}\left|z_{0}-\dot{q}\right|^{5 / 6} \operatorname{sign}\left(z_{0}-\dot{q}\right)+z_{1}, \\
& \dot{z}_{1}=v_{1}, \\
& v_{1}=-\lambda_{1} L^{1 / 5}\left|z_{1}-v_{0}\right|^{4 / 5} \operatorname{sign}\left(z_{1}-v_{0}\right)+z_{2}, \\
& \dot{z}_{2}=v_{2}, \\
& v_{2}=-\lambda_{2} L^{1 / 4}\left|z_{2}-v_{1}\right|^{3 / 4} \operatorname{sign}\left(z_{2}-v_{1}\right)+z_{3}, \\
& \dot{z}_{3}=v_{3}, \\
& v_{3}=-\lambda_{3} L^{1 / 3}\left|z_{3}-v_{2}\right|^{2 / 3} \operatorname{sign}\left(z_{3}-v_{2}\right)+z_{4}, \\
& \dot{z}_{4}=v_{4}, \\
& v_{4}=-\lambda_{4} L^{1 / 2}\left|z_{4}-v_{3}\right|^{1 / 2} \operatorname{sign}\left(z_{4}-v_{3}\right)+z_{5}, \\
& \dot{z}_{5}=v_{5}, \\
& v_{5}=-\lambda_{5} L \operatorname{sign}\left(z_{5}-v_{4}\right) .
\end{aligned}
$$

The initial value $z_{0}=0.1 ; \lambda_{0}=\lambda_{1}=\lambda_{2}=\lambda_{3}=\lambda_{4}=$ $\lambda_{5}=50$; the initial values $z_{1}, z_{2}, z_{3}, z_{4}, z_{5}=0 ; L=1400$; the simulation step length is 0.0001 seconds. The simulation results are shown in Figure 23.

As the figure shows, the fifth-order differentiator has a rather ideal tracking effect on the third-order differential tracking, the tracking error being only about 0.0005 (see Table 6).

To sum up, the Arbitrary-Order Robust Exact Differentiator can estimate rather ideal differential signals by selecting
TABLE 7

\begin{tabular}{lc}
\hline Length & $3.65 \mathrm{~m}$ \\
$L_{\text {ref }}$ & $3.65 \mathrm{~m}$ \\
$X_{G}$ & $1.77 \mathrm{~m}$ \\
$S_{\text {ref }}$ & $0.026 \mathrm{~m}^{2}$ \\
Diameter & $0.178 \mathrm{~m}$ \\
Mass & $101.6 \mathrm{Kg}$ \\
$I_{Z}$ & $106.3 \mathrm{Kg} \cdot \mathrm{m}^{2}$ \\
\hline
\end{tabular}

an appropriate sampling interval and using the differentiator with a relatively high number of orders.

\section{Physical and Geometric Characteristics}

See Table 7.

\section{Conflict of Interests}

The authors declare that there is no conflict of interests regarding the publication of this paper.

\section{References}

[1] P. Zarchan, Tactical and Strategic Missile Guidance, 2002.

[2] D. Zhou, C. Mu, and W. Xu, "Adaptive sliding-mode guidance of a homing missile," Journal of Guidance, Control, and Dynamics, vol. 22, no. 4, pp. 589-594, 1999.

[3] G. Zeng and M. Hu, "Finite-time control for electromagnetic satellite formations," Acta Astronautica, vol. 74, pp. 120-130, 2012.

[4] S. R. Kumar, S. Rao, and D. Ghose, "Nonsingular terminal sliding mode guidance with impact angle constraints," Journal of Guidance, Control, and Dynamics, vol. 37, no. 4, pp. 1114-1130, 2014.

[5] Y. Liang, C. Chen, D. Liaw, Y. Feng, C. Cheng, and C. Chen, "Robust guidance law via integral-sliding-mode scheme," Journal of Guidance, Control, and Dynamics, vol. 37, no. 3, pp. 1038-1042, 2014.

[6] A. V. Savkin, P. N. Pathirana, and F. Faruqi, "Problem of precision missile guidance: LQR and $H_{\infty}$ control frameworks," IEEE Transactions on Aerospace and Electronic Systems, vol. 39, no. 3, pp. 901-910, 2003.

[7] H. Y. Chen and C.-C. Yang, "Nonlinear $\mathrm{H}_{2} / \mathrm{H}_{\infty}$ guidance design for homing missiles," in Proceedings of the AIAA Guidance, Navigation, and Control Conference and Exhibit, vol. 77, 2001.

[8] P. K. Menon, G. D. Sweriduk, and E. J. Ohlmeyer, "Optimal fixed-interval integrated guidance-control laws for hit-to-kill missiles," in Proceedings of the AIAA Guidance, Navigation, and Control Conference and Exhibit, AIAA 2003-5579, Austin, Tex, USA, August 2003.

[9] S. S. Vaddi, P. K. Menon, and E. J. Ohlmeyer, "Numerical SDRE approach for missile integrated guidance-control," in Proceedings of the AIAA Guidance, Navigation, and Control Conference and Exhibit, AIAA 2007-6672, Hilton Head, SC, USA, August 2007.

[10] M. Xin, S. N. Balakrishnan, and E. J. Ohlmeyer, "Integrated guidance and control of missiles with Theta-D method," IEEE 
Transactions on Control Systems Technology, vol. 14, no. 6, pp. 981-992, 2006.

[11] T. Shima, M. Idan, and O. M. Golan, "Sliding-mode control for integrated missile autopilot guidance," Journal of Guidance, Control, and Dynamics, vol. 29, no. 2, pp. 250-260, 2006.

[12] Y. B. Shtessel and C. H. Tournes, "Integrated higher-order sliding mode guidance and autopilot for dual control missiles," Journal of Guidance, Control, and Dynamics, vol. 32, no. 1, pp. 79-94, 2009.

[13] F. Y. Dong, H. M. Lei, and C. J. Zhou, "Integrated robust high order sliding mode guidance and control for missiles," Acta Aeronautica et Astronautica Sinica, vol. 34, no. 9, pp. 2212-2218, 2013.

[14] H. Mingzhe and D. Guangren, "Integrated guidance and control of homing missiles against ground fixed targets," Chinese Journal of Aeronautics, vol. 21, no. 2, pp. 162-168, 2008.

[15] A. Levant, "Homogeneity approach to high-order sliding mode design," Automatica, vol. 41, no. 5, pp. 823-830, 2005.

[16] Y.-W. Liang, C. Chen, D. Liaw, Y. Feng, C. Cheng, and C. Chen, "Robust guidance law via integral-sliding-mode scheme," Journal of Guidance, Control, and Dynamics, vol. 37, no. 3, pp. 1038-1042, 2014.

[17] L. Wang, Y. Sheng, and X. Liu, "Continuous time-varying sliding mode based attitude control for reentry vehicle," Proceedings of the Institution of Mechanical Engineers Part G: Journal of Aerospace Engineering, 2014.

[18] A. Levant, "Higher-order sliding modes, differentiation and output-feedback control," International Journal of Control, vol. 76, no. 9-10, pp. 924-941, 2003.

[19] A. Levant, "Quasi-continuous high-order sliding-mode controllers," IEEE Transactions on Automatic Control, vol. 50, no. 11, pp. 1812-1816, 2005. 


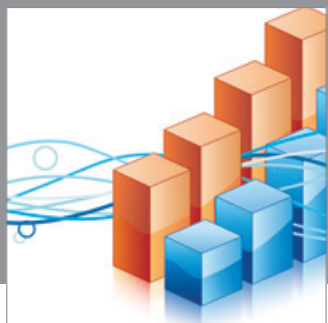

Advances in

Operations Research

mansans

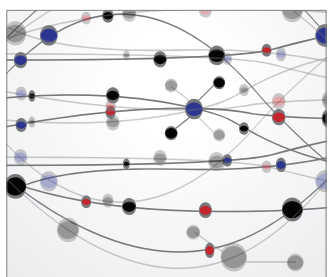

The Scientific World Journal
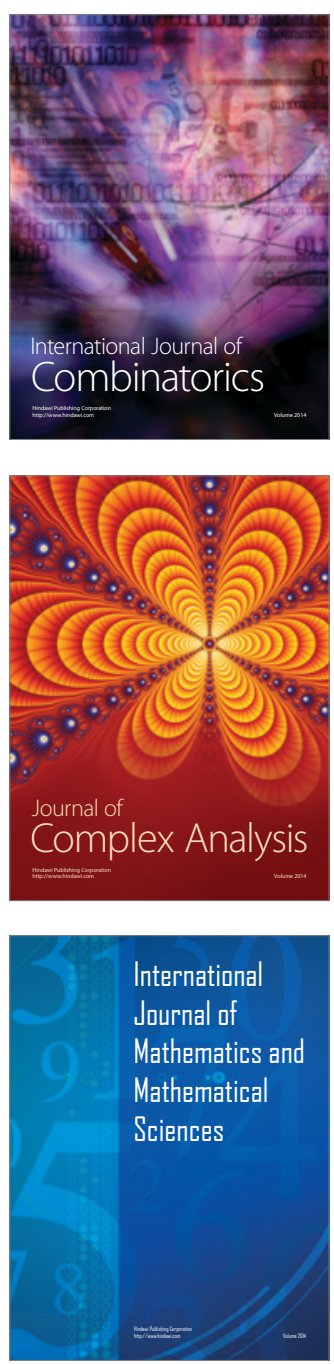
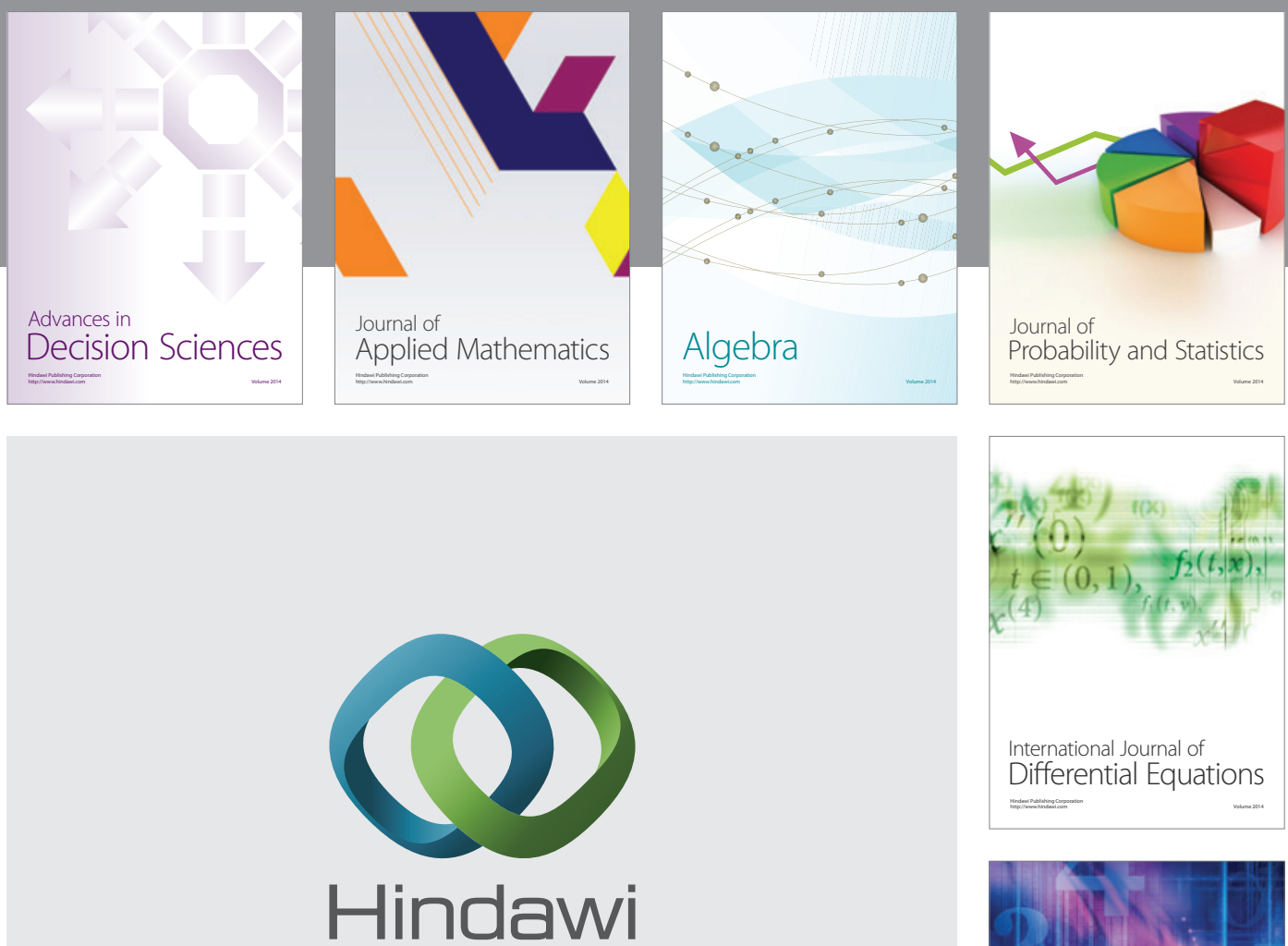

Submit your manuscripts at http://www.hindawi.com
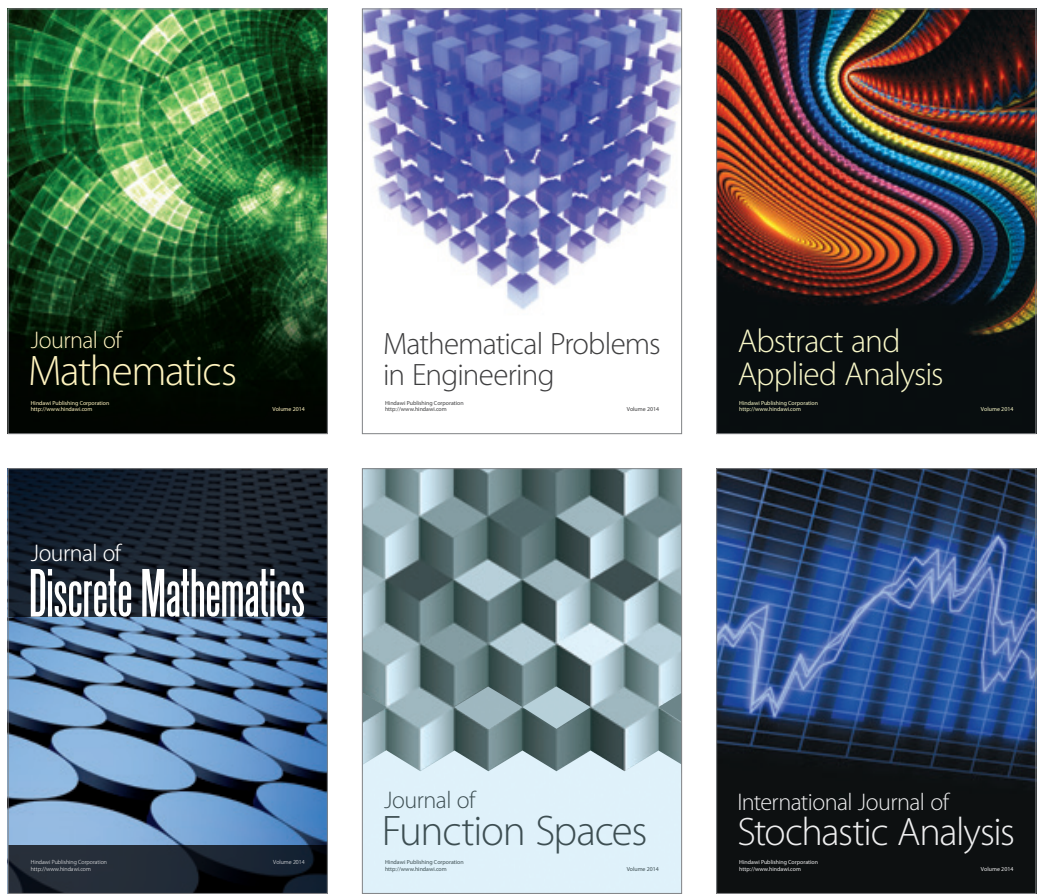

Journal of

Function Spaces

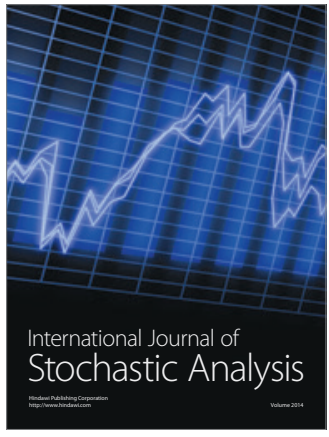

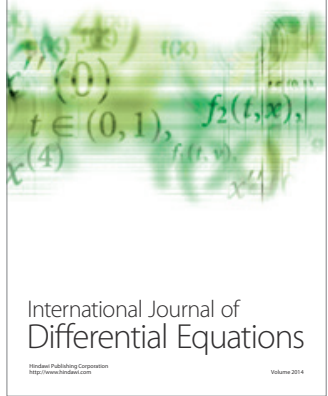
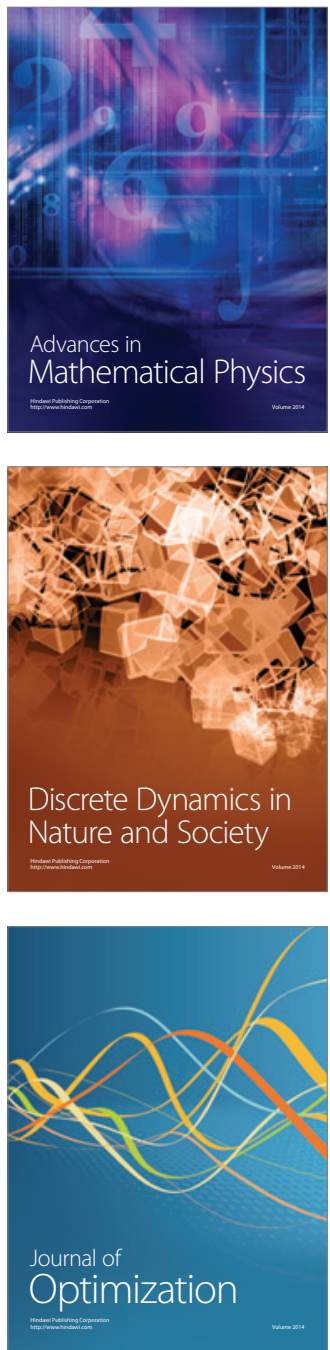